XLVI.

\title{
Phosphorsäure- und Kalkstoffwechsel bei Osteomalacie unter dem Einfluss der Phosphortherapie.
}

\author{
Von
}

Gerhard Hotz,

Assistenzarzt an der chirurgischen Klinik des Basler Burgerspitals.

Basel und die kleineren Thäler seiner Umgebung sind bekannt als ein Sitz der endemischen Osteomalacie; deshalb gehört das Bild dieser Krankheit auf unseren Kliniken nicht zu den Seltenheiten.

Im Jahre 1903 befanden sich gleichzeitig 2 osteomalacische Frauen auf der medicinischen Klinik. Herr Professor W. His veranlasste mich, den Calcium- und Phosphorsäurestoffwechsel dieser beiden Fälle zu untersuchen; vor Allem sollte der Einfluss der Phosphortherapie auf die Osteomalacie festgestellt werden. Die vorliegende Arbeit bildet demnach eine Fortsetzung der Untersuchungen, welche Herr Professor His durch Sauerbruch an einem Falle von juveniler Osteomalacie auf der Leipziger Klinik vornehmen liess.

Für die Anregung und das Interesse, welches Herr Professor His der Arbeit entgegenbrachte, für die vielseitige Unterstützung, welche ich durch die Herren Assistenzärzte Dr. Stähelin und Dr. Bloch erfahren durfte, möchte ich an dieser Stelle meinen verbindlichsten Dank aussprechen.

\section{Krankengeschichte und Status.}

1. Frau Rosine Gasser, geboren 1863 in Basel, angeblich als Frühgeburt im 8. Monat. Vater gestorben an Lungentuberculose, Mutter und ein Bruder gesund. 3 Geschwister starben im Säuglingsalter an Gichtern und Brechruhr; ein Kind an Lebensschwäche, das andere im 12. Jahre an Spondylitis tuberculosa. Pat. verbrachte die Jugend unter guten Verhältnissen im Elternhause. Als Kind Pneumonie, Scharlach. Menarche mit 16 Jahren, regelnässig, stark; während der Periode fühlte sie sich oft sehr schwach, Müdigkeit im Rücken und in den Beinen. Mit 27 Jahren Geburt eines ausgetragenen Kindes. Im Wochenbett erkrankte sie an Influenzapneumonie und kam deshalb auf die medicinisohe Klinik.

Die Krankengeschichte schildert sie als bleiches, decrepides Individuum; keine Angaben, welche für Osteomalacie sprechen. Das Kind wurde nicht gestillt. In den folgenden 2 Jahren bewohnto sie ein feuchtes Hinterhans, wurde 29 jährig durch eine schwierige Extraction bei Steisslage von einem ebenfalls reifen Kinde entbunden. Während der Schwangerschaft noch keine Störungen. Aus dem Wochenbett erhob sie sich mit einer mehrere Monate andauernden Schwäche und Schmerzen im Rücken. 
Das Stillgeschäft musste nach 3 Wochen wegen Mastitis unterbrochen werden. Der Gang blieb längere Zeit schworfällig; doch verloren sich dio Beschwerden später vollständig. Erst nach 6 Jahren traten Schmerzen im Rücken auf, welche als Rheumatismus behandelt wurden. "3 Jahre später bemerkte Pat., dass ihre Gestalt zusammengesunken sei, die Röcke waren zu lang geworden. Andauernde Schmerzen in der Rückenwirbelsäule, im Thorax und im Becken, dazu grosse Müdigkeit und Schwäche in den Beinen, so dass sie oft glaubte, zusammenbrechen zu müssen. Der Rücken wurde gebeugt, der Thorax verbogen. So schleppte sich die Frau, wenn auch nie bettlägerig, so doch an's Haus gebannt, bis zum März 1903 hin. Schliesslich konnte sie nur noch, auf Stühle gestützt, sich im Zimmer umher bewegen. Die Schmerzen strahlen vom Rücken nach den Armen, in den Brustlorb und gegen das Becken zu aus. Häufige Krämpfe in den Extensoren der Oberschenkel. Die Menses verursachen stets vermehrte Beschwerden; seit 3 Monaten sind sie ausgeblieben, ohne dass Gravidität besteht. Am 13. März 1903 wurde Pat. auf die medicinische Klinik aufgenommen.

Status: Grösse $126 \mathrm{~cm}$. Gewicht 30,6 kg. Schlechter Ernährungszustand. Haut trocken, schlaff, in den Gelenkbeugen auffallend dunkel gefärbt. Haare dunkel, dicht stehend, trocken, sehr dünn. Augen: Pupillen reagiren prompt; links Strabismus convergens. Zunge nicht belegt. Zähne sehr defect. Schilddrüse klein. An den grossen Halsvenen diastolische Pulsation. Wirbelsäule: der thoracale Abschnitt bildet einen gleichmässig gewölbten, im oberen Theil nach rechts, im unteren nach links ausgebogenen Buckel. Der Halstbeil ist im 6 . Wirbel stark nach vorn geknickt, so dass das Kinn an die Brust angedrückt wird, und der Kopf nur wenig seitlich bewegt werden kann. Beide Schulterblätter stehen nach aussen ab. Die rechte Thoraxseite ist weiter als die flache linke. Die untere Apertur scheint zusammengeschnürt und steht so tief im Becken, dass die Cristae ilei den Rippenbogen 2 Fingerbreit überragen.

Die Lungen geben einen der Thoraxdifformität entsprechenden Schallunterschied. Athmung vesiculär, in den unteren Partien klingendo Rasselgeräusche. Die absolute Herzdämpfung reicht bis zur vorderen Axillarlinie. Hier findet man im 4. Intercostalraum den Spitzenstoss. Ueber der Herzspitze leises systolisches Geräusch. 2. Pulmonalton verstärkt. Puls 85 pro Minute, regelmässig, klein. Temperatur afebril. Das Abdomen zeigt einen mässigen Hängebauch. Distanz zwischen Schwertfortsatz und Symphyse $20 \mathrm{~cm}$. Am Becken fühlt man einen stark schnabelförmigen Symphysenvorsprung, die Spinae sind nach aussen umgebogen. Maasse: Dist. cristarum $21 \mathrm{~cm}$, spinarum $20^{1} / 2 \mathrm{~cm}$, Baudelocque $19^{1} / 2 \mathrm{~cm}$, Tuber isch. $5 \mathrm{~cm}$. Schambogen sehr eng, lässt kaum 1 Finger einführen. Uterus klein, anteflectirt. Druck auf die Darmbeinschaufeln schmerzhaft. Das Becken federt deutlich, ebenso der Thorax. Die Arme können gestreckt nicht ephoben werden, Knarren und Schmerzen im Schultergelenk. Deutliche Difformitäten dieser Knochen bestehen nicht. Die Beine werden gestreckt kanm über der Unterlage gehalten. Ab- und Adduction sehr schwach. Patellarreflexe beiderseits gesteigert. Das Stehen und Gehen ist mit grossen Schmerzen im Rücken und im Becken verbunden; die Frau ist deshalb zu vollkommener Bettruhe gezwungen; doch klagt sie auch in dieser Lage über quälendo Knochenschmerzen. Im Urin weder Eiweiss noch Zucker.

Im Verlauf der Spitalbehandlung lassen die Schmerzen bald nach. Zwei Wochen nach dem Eintritt ist bereits eine zweifellose Besserung im subjectiven Befinden zu constatiren. Die Vorperiode des Stoffwechselversuches beginnt am 26. März. Am 9. April setzt die Phosphortherapie ein. In Form von Emulsio Kassowitz werden täglich $2 \mathrm{Mal} \mathrm{1/2} \mathrm{mg}$ Phosphor gegeben. Nach 11 Tagen sind Schmerzen und Druckempfindlichkeit der Knochen wesentlich vermindert; die Frau kann sich ohne Beschwerden aufsetzen, die Beine werden mit grösserer Kraft und Ausdauer gehoben. Die Entziehung des Phosphors in den folgenden 10 Tagen der Nachperiode bedingt keine Aenderung des Znstandes, Jedenfalls tritt keine sichtliche Verschlimmerung 
ein. Ebenso verhält es sich später bei der Darreichung von Thyreoideatabletten. Vom 8. Mai an wird wiederum Phosphor gegeben. Nun eilt die Genesung rasch vorwärts, so dass die Osteomalacische in kurzer Zeit das Bett verlässt und sich bei kleinen Zimmerdiensten betheiligen kann. Nach zwei weiteren Monaten sind alle Schmerzen geschwunden, die Knochen nicht mebr druckempfindlich. Am 5. September verlangt die Frau ihre Entlassung nach Hause. Sie ist den ganzen Tag über auf, sehr regșam und hält sich für kräftig genug, um ihre Hausgeschäfte besorgen zu können. Der osteomalacische Process ist somit zum Stillstand gekommen, die Krankheit erscheint ausgeheilt, wenn auch ihre deformirenden Einwirkungen auf das Skelett nicht mehr ausgeglichen werden konnten. Während des Spitalaufenthaltes hat die Frau ca. 0,13 g Phosphor eingenommen. Eine dieser Therapie zuzuschreibende Störung im Allgemeinbefinden ist nicht aufgetreten. Das Körpergewioht stieg bis zum Ende des Versuches auf $34,3 \mathrm{~kg}$, bis zur Entlassung auf $36,7 \mathrm{~kg}$.

Diese Besserung hielt zu Hause mehrere Monate lang an. Dann stellten sich aber allmälig die alten Beschwerden wieder ein. Die Muskelkraft erlahmte, die Bewegungsfreiheit wurde durch Schmerzen eingeschränkt. So treffen wir die Frau im Frühjahr 1904 wieder auf der Klinik. Diesmal hatte sie das Krankenhaus früher aufgesucht, ihr Allgemeinbefinden erreichte bei Weitem nicht den traurigen Zustand wie bei der ersten Aufnahme. Die wiederum angewandte Phosphortherapie erzielte in 4 Wochen die Wiederherstellung, ein bis jetzt andauerndes Wohlbefinden, da sich die Frau an die Weisung hält, auch zu Hause ihre Phosphoremulsion zu gebrauchen.

2. Frau Gehrig, geboren 1842 in Basel. Eltern an unbekannter Krankheit gestorben. Ein Bruder und eine Schwester litten Jahre lang an "Gliedersucht". Etwas Näheres ist über diese Krankheit nicht zu erfahren. Pat. lebte stets in ärmlichen Verhältnissen. Mit 23 Jahren Typhus, mit 27 Jahren Magenkrämpfe, welchen sich eine "Bauchfellentzündung" anschloss. Schon mit 20 Jahren will Pat. an "Gliederschmerzen" gelitten haben, welche sich später oft wiederholten. Damals waren besonders die Gelenke der oberen Extremitäten betroffen. Drei leichte Geburten mit 35, 37 und 40 Jahren. In der letzten Schwangerschaft stellten sich vom 2. Monate ab Schmerzen im Kreuz, Rücken und in beiden Beinen ein. Sie will jenes Mal kleiner geworden sein. Die letzte Geburt ging leicht von Statten. Dauer der Wehen bis zur Austreibung 7 Stunden. Das Kind sei allerdings sehr klein gewesen und bald gestorben. Die Knochenschmerzen verschlimmerten sich nach dem Wochenbette, während Pat. stillte, etwa 2 Monate lang. Dann trat sehr langsame Besserung ein; es dauerte aber über $1 \mathrm{Jahr}$, bis sie wieder ordentlich gehen konnte, um als Wäscherin zu arbeiten. 9 Jahre lang war sie gesund, bis zum Eintritt der Menopause. Nun stellten sich die früheren Schmerzen im Kreuz, Rücken und Becken wieder ein; Anfangs verschwanden sie jeweilen im Sommer, im Winter wurde es regelmässlg schlimmer, schliesslich versagte die Kraft zur Arbeit. $1898 \mathrm{kam}$ sie zum ersten Mal in Spitalbehandlung, ihr Leiden wurde, als Ischias aufgefasst. $1900 \mathrm{kam}$ sie auf die chirurgische Klinik unseres Bürgerspitals. Hier wurde die Osteomalacie erkannt und die Frau auf die medicinische Klinik verlegt. : Der damalige Aufnahmebefund entspricht ungefäbr dem später mitzutheilenden Status. Nun folgen in kurzer Zeit mebrere Spitalaufenthalte. Die Frau wurde abwechselnd mit Soolbädern, Schwitzcuren, Kalk, Phosphorpräparaten, mit Thyreoidea- und Ovarialtabletten behandelt. Der Erfolg war stets nur scheinbar und von kurzer Dauer. Emulsio Kassowitz, allerdings nie über Monate dargereicht, brachte nur geringe Besserung, am meisten Linderung verschaffte Aspirin. Im Sommer 1902 verweilte sie auf der Frauenklinik. Die Castration wurde damals von Herrn Professor v. Herff abgelehnt, erstens in Børücksichtigung der bereits vor 11 Jahren eingetretenen Menopause und vor allem, weil sich die Frau in einem sehr decrepiden Zustande befand. Im Januar 1903 erneute Verschlimmerung, 
so dass Pat. unfähig, sich zu rühren, an's Bett gefesselt wurde; auch in gestreckter Lage wurde sie von beständigen Knochenschmerzen gequält. In diescm Zustande kam sie am 30. März wieder auf die medicinische Klinik.

Status: Grösse $138 \mathrm{~cm}$, Gewicht $43 \mathrm{~kg}$. Blasse, magereFrau. Augenbewegungen, Pupillenreaction normal. Die Zähne fehlen alle. Schilddrüse nicht vergrössert. Halswirbelsäule lordotisch, Hinterhaupt in den Nacken gepresst. Die Rückenwirbelsäule bildet einen spitzen, stark gekrümmten Buckel in der oberen Hälfte. Thorax tief. Oberer Theil des Sternurns stark vorspringend, unterer eingezogen. Die Rippenbogen stehen innerhalb der breit auseinanderweichenden Darmbeinkämme. Der Lungenbefund ergiebt keine abnormen Verhältnisse. Athmung mühsam, stets mit Schmerzen der Rippen verbunden. Herz: geringe Verbreiterung der absoluten Dämpfung nach links. Spitzenstoss in der Mamillarlinie im V. Intercostalraum. Ueber der Spitze kurzes systolisches Geräusch mit leichter Verstärkung des II. Pulmonaltones. Puls 70 pro Minute, regelmässig, klein. Kein Fieber. Es besteht ein Hängebauch. Die Leberdämpfung überragt den Rippenbogen fingerbreit; in der Gegend der Gallenblase geringe Druckempfindlichkeit. Becken breit, Symphyse schnabelförmig, sehr druckempfindlich.

Maasse: Dist. spinar. 24, cristar. 29, trochanter. 271/2, Baudelocque $20 \mathrm{~cm}$. Musculatur des Oberschenkels auffallend schwach entwickelt. Active Flexion des Oberschenkels kaum möglich. Ab- und Adduction mit ganz geringer Kraft ausgeführt. Bei passiven Bewegungen heftige Schmerzen im ganzen unteren Skelettheil. Patellarund Achillessehnenreflex beiderseits lebhaft. Andeutnng von Fussclonus. Thorax, Wirbelsäule, Becken, alle Knochen der Extremitäten sind in hohem Grade druckempfindlich, selbst die Erschütterung des Bettes beim Gehen im Zimmer verursacht Schmerzen. Die Sensibilität ist überall normal, als einzige Störung findet man in der Höhe des Nabels eine handbreite gürtelförmige Zone von Kältehyperästhesie.

Am 1. April Beginn des Stoffwechselversuches, vom 9. April ab wurde Emulsio Kassowitz gegeben, täglich $1 \mathrm{mg}$ Phosphor. Am 21. April Ansetzen der Phosphortherapie, vom 28. April bis zum 5. Mai erhielt Pat. Thyreoideatabletten. Der Anfangs kaum erträgliche Zustand besserte sich 8 Tage nach Beginn der Phosphordarreichung so weit, dass Pat. wenigstens quallos zu Bette liegen konnte. Eine bemerkenswerthe Aenderung ist während der späteren Zeit des Stoffwechselrersuches nicht zu verzeichnen; erst 5 Wochen nach Beendigung desselben, nach längerer Phosphoreinnahme, hat sich die Frau so weit erholt, um sich selbst im Bette aufrichten zu können. Der Zustand ist immer noch kläglich genug. Von Mitte August ab werden Ovarialtabletten gegeben. Inzwischen nimmt die Krankheit eine etwas günstigere Wendung; die Knochenschmerzen lassen nach, Pat. verlässt das Bett und bewegt sich erst mit Krücken, später mit Stöcken im Zimmer umhor. Am 20. September wird sie auf ihren dringenden Wunsch nach Hause entlassen, sie selbst ist von dem Nutzen der Ovarialtabletten überzeugt. Ihr Körpergewicht war während des Stoffwechselversuches gleich geblieben, später ergab sich eine Abnahme von $3 \mathrm{~kg}$. Ein Jahr später, im Sommer 1904, kam die Frau wieder zur Aufnahme in einem bedenklich elenderen Zustande als zur Zeit des Stoffwechselversuches. Die Gestalt war um $5 \mathrm{~cm}$ eingesunken, das Körpergewicht um $10 \mathrm{~kg}$ gefallen. Es besteht ausgesprochene Kachexie. Die Phosphortherapie erwies sich als wirkungslos. Lange Pflege, Thyreoidea- und Ovarialtabletten, welche später gegeben wurden, gestalteten die Lage wieder erträglicher und hoben die Rewegungsfähigkeit. Die Frau verlangte wiederum, entlassen zu werden und erlag im Herbst 1904 zu Hause einer Lungencomplication. 
Im ersten Fall handelt es sich um eine 40 jährige Frau im floriden Stadium der Osteomalacie. Die Krankheit wird unter dem Einfluss der Spitalbehandlung und der Phosphortherapie zum Stillstand gebracht, die Frau wird geheilt. Im zweiten Fall treffen wir eine 61 jährige, weit vorgeschrittene Osteomalacische. Das Leiden wird durch die gleichen Heilfactoren nur wenig und vorübergehend gebessert. Die Krankheit führt nach kurzer Zeit zum Tode.

In der vorliegenden Arbeit soll der Kalk- und Phosphorstoffwechsel dieser beiden klinisch verschieden verlaufenden Fälle festgestellt und untersucht werden, einmal, wie die Darreichung des anorganischen Phosphors die Kalk- und Phosphorbilanz beeinflusst und zweitens, ob diese Beeinflussung den klinischen Beobachtungen des Krankheitsverlaufes entspricht. Daraus wird ein Beitrag zur Kenntniss der Osteomalacie und zur Beurtheilung des Werthes der Phosphortherapie gewonnen werden, der mit den Erfahrungen anderer therapeutischer Maassnahmen verglichen werden darf.

Dem Stoffwechselversuch liegt folgender Plan zu Grunde: Während einer Vorperiode wurde der Kalk- und Phosphorumsatz ohne Beeinflussung" durch irgendwelche medicamentöse Therapie festgestellt. Diese Vorperiode betrug für Frau Gasser 10, für Frau Gehrig 8 Tage. Ihr schloss sich sofort die Phosphorperiode an, während welcher täglich $1 \mathrm{mg}$ Phosphor als Emulsio Kassowitz gegeben wurde; sie erstreckt sich im ersten Falle über 11, im zweiten über 12 Tage, dann wurde die Phosphordarreichung wieder ausgesetzt. Diese Nachperiode dauerte 10, resp. 7 Tage. Zuletzt wurde eine Thyreoideaperiode angeschlossen. Die Frauen erhielten während 8 Tagen täglich 3 Pastilli c. Thyreoidin. sicc., ein Präparat von Hoffmann La Roche; jede Tablette enthält $0,3 \mathrm{~g}$ frische Schilddrüsensubstanz. Der ganze Versuch, der bei beiden Patienten gleichzeitig durchgeführt wurde, so dass die nämlichen Nahrungsmittel zur Vertheilung gelangten, umfasst also bei Frau Gasser 39, bei Frau Gehrig 35 Tage.

Die Kost war gemischt, abwechslungsreich und doch in Bezug auf Calcium- und Phosphorgehalt für die einzelnen Perioden ziemlich gleichwerthig.

An festen Speisen wurde gegeben: Hackbraten, Kalbsbraten, Beefsteak ca. 60-120 g täglich. Vom Fleisch wurde jeweilen ein grösseres Stück auf einige Tage vertheilt. Das Zugemüse wechselte täglich zwischen Nudeln, Macaroni, Reis, je $50 \mathrm{~g}$ Trockensubstanz zur Speise verwendet. Kartoffelpurée, Apfelmus $200-400 \mathrm{~g}$, feines Brot $70-140 \mathrm{~g}$, Butter 15-30 g, Johannisbeergelée. Zu Suppen wurden verschiedene Maggipräparate verwendet. Die Milch, 600-1200 cem täglich, lieferte der hiesige allgemeine Consumverein. Hierbei war angeordnet, dass jeweilen eine grössere Flaschenzahl aus demselben Stammgefäss abgefüllt wurde. Diese unter dem Namen "sterilisirte Kindermilch" in den Handel gebrachte Milch hielt sich während 10 Tagen vollkommen gut. Als Getränk wurde ausserdem Passugger Ulricusquelle 350-700 cem gegeben. Alle Speisen, zu deren Zubereitung Wasser nöthig ist, wie Nudeln, Kaffee etc., wurden mit Aqua dest. hergestellt. Von Nudeln, Macaroni, 
Reis wurde das abgegossene Kochwasser aufbewahrt, analysirt und bei der Berechnung der Speise in Abzug gebracht. Von allen Nahrungsmitteln wurde eine Portion vor der Vertheilung zur Analyse aufgehoben, frisch gewogen, dann über dem Wasserbad und im Trockenofen bis zur Gewichtsconstanz getrocknet, wiederum gewogen und in pulverisirtem Zustand in dicht verschlossenen Flaschen zur Untersuchung aufbewahrt. Von jeder Milchlieferung wurden einige Flaschen zurückbehalten.

Tabelle I zeigt die gefundenen Analysenwerthe, den $\mathrm{P}_{2} \mathrm{O}_{5}$ - und $\mathrm{CaO}$ Gehalt der frischen Nahrungsmittel in Procenten.

Als Beispiel der täglichen Nahrungsaufnahme führe ich den 17. April, den zufällig gewählten 10. Tag der Phosphorperiode an.

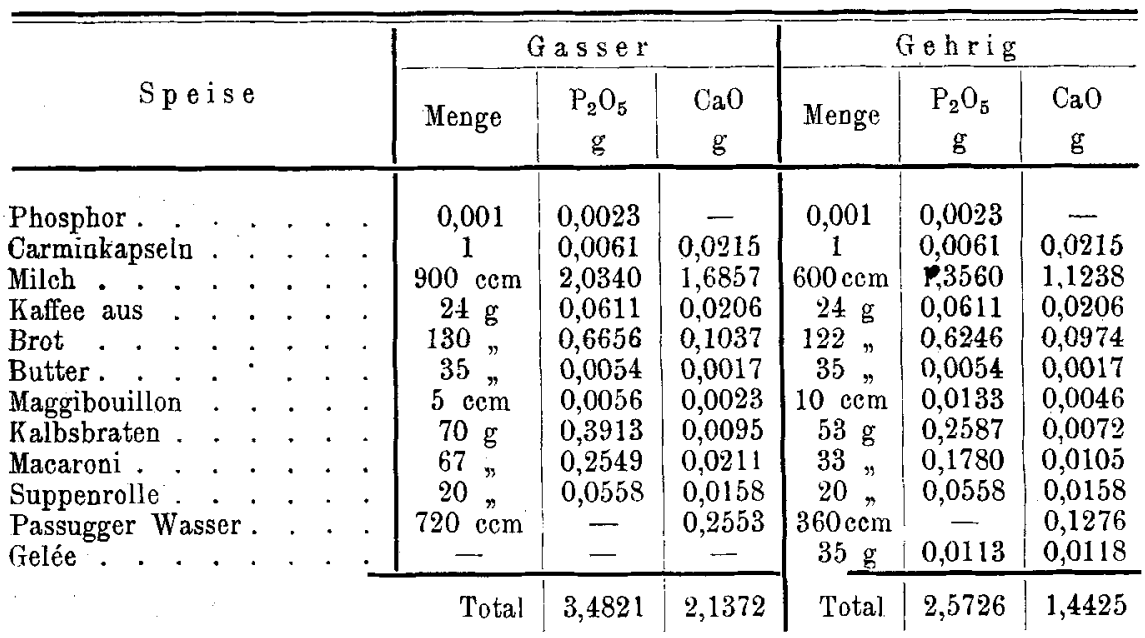

In Calorien beträgt die tägliche Nahrungsaufnahme für Frau Gasser durchschnittlich etwa 1900, für Frau Gehrig etwa 1600; pro Kilogramm Körpergewicht 60 resp. 35 Calorien pro 'lag. Die erste Patientin nimmt bei dieser Kost an Körpergewicht zu, die andere hält sich kaum auf ihrer Höhe.

Die Ausgaben wurden aus dem gesammelten Harn und Koth bestimmt. Die 24 stündige Urinmenge wurde gemessen, zur Verhinderung der Zersetzung und des Ausfallens von Sediment mit $20 \mathrm{ccm}$ verdünnter $\mathrm{HCl}$ versetzt und von jedem Tag eine grosse Probe aufbewahrt. Der Koth wurde bis zur Gewichtsconstanz getrocknet; besonders sorgfältig wurde auf eine genaue Abgrenzung desselben geachtet. Um diese zu erreichen, wurden Morgens, 1 Stunde vor dem ersten Frühstück. $1 g$ haltige Carmin- oder Kohlekapseln gegeben. Der Stuhl war in der Regel geformt, so dass sich die einzelnen Schichten gut abtheilen liessen. Während der Vorperiode wurde der Koth der ersten 6 Tage vereinigt. später alle 2 Tage abgegrenzt und bestimmt.

Für den Versuch bei Frau Gasser sind wir uns keines Verlustes bewusst; bei Erau Gehrig sind leider 3 Urinproben vor der Bestimmung weggegossen worden, diejenige rom ersten und letzten 'Tag der Phosphordarrejchung und die vom zweiten Tag der Nachperiode. Einnahmen und Koth sind vollständig. Es wurde versucht, den entstandenen Verlust 
bei der Berechnung auszugleichen dadurch, dass für den weggegossenen Harn der Mittelwerth des dem Verlusttage vorausgegangenen und nachfolgenden Tages eingesetzt wurde. Die CaO-Ausscheidung im Harn ergiebt ungefähr den zehnten Theil der im Koth ausgeführten Menge; es kann sich bei der versuchten willkürlichen Berechnung deshalb höchstens um einen Fehler von einigen Centigrammen handeln, und ein solcher kommt für die Bilanz der einzelnen Perioden nicht in Frage. Ungünstiger liegt die Sache für die Berechnung der $\mathrm{P}_{2} \mathrm{O}_{5}$-Ausscheidung. Der Harngehalt ist gross und schwankt beträchtlich, demnach vermögen die angenommenen Werthe, die sich an Hand der bekannten Urinmenge und des spec. Gewichtes einigermaassen controliren lassen, die Gesammtresultate nicht wesentlich zu ändern.

Die Analysen wurden nach folgendem Verfahren ausgeführt: Veraschung nach der bekannten Neumann'schen Methode ${ }^{1}$ ) durch Salpetersäure-Schwefelsäuregemisch im erhitzten Jenenserkolben. Aus der rückständigen Flüssigkeit wurde auf gewichtsanalytischem Wege bestimmt:

a) Die $\mathrm{P}_{2} \mathrm{O}_{5}$ durch Fällung mit molybdensaurem Ammon, Lösung des ausgewaschenen Niederschlages in $\mathrm{NH}_{3}$ und nachheriger Fällung mit Magnesiamixtur, geglüht als $\mathrm{Mg}_{2} \mathrm{P}_{2} \mathrm{O}_{7}$.

b) Der Kalk als CaO durch Fällung mit Ammonoxalat und nachherigem Glühen.

Vom Trockenkoth wurde ca. $1 \mathrm{~g}$, von den Nahrungsmitteln mit geringem $\Lambda$ schegehalt bis zu $10 \mathrm{~g}$ verwendet. Für die Phosphorbestimmung im Harn wurden 20, zur Kalkberechnung $100 \mathrm{ccm}$ erst eingedampft und dann mit Säuregemisch verascht. Von jeder Probe wurden 2, nöthigenfalls mehr Controlanalysen ausgeführt.

Die Resultate der beiden Stoffwechselversuche finden sich in den nachfolgenden Tabellen zusammengestellt:

Tabelle I giebt die eigenen Analysen der Nahrungsmittel; die drei folgenden beziehen sich auf den Fall Gasser.

Tabelle II. Menge, spec. Gewicht, $\mathrm{P}_{2} \mathrm{O}_{5}$ - und CaO-Gehalt im Harn und Koth für die einzelnen Tage.

Tabelle III. Tagesbilanz. Die Einnahmen, nach dem im Beispiel angeführten Modus berechnet, werden im Einzelnen nicht angegeben.

Tabelle IV. Calcium- und Phosphorbilanz der 4 Perioden.

Im ersten Versuch ergiebt also die Kalkbilanz in der Vorperiode eine Retention von. . . . 0,6023 g; pro Tag 0,0602 g Phosphorperiode eine Retention von. . 2,2777 n. " 0,2070 " Nachperiode eine Mehrausscheidung von 0,5236" " " $" 0,0523 "$ Thyreoideaperiode eine Retention von . 0,1026" " " 0,0128"

Die geringe Retention der Vorperiode spricht dafür, dass der osteomalacische Process, die Halisteresis, wohl in Folge der allgemein

1) Albert Neumann, Einfache Veraschungsmethode. Zeitschr. f. physiol. Chemie. Bd. 37. 1902. S. 115. 
günstigeren Lebensbedingung, der Bettruhe und Krankenhauspflege zum Stillstand gekommen ist, dass sich sogar bereits ein Kalkansat $z$ geltend macht. Hierzu stimmt auch die Angabe der Krankengeschichte, dass bereits vor Finsetzen der Phosphortherapie eine merkliche Besserung im allgemeinen Befinden auftrat; die Knochenschmerzen liessen nach. Die Phosphortherapie bewirkt klinisch eine bei dieser Frau mehrfach erprobte und in die Augen springende günstige Wirkung auf den Krankheitsverlauf. Die Beschwerden treten rasch zurück, die Beweglichkeit stellt sich mit der wachsenden Kraft wieder ein. Dieser eclatante Erfolg lässt eine Aenderung des Stoffwechsels im Sinne eines Kalkansatzes erwarten. Die Bilanz zeigt uns denn auch eine erhebliche Retention, mehr als 10 pCt. des eingeführten Gesammtkalks werden rom Körper zurückgehalten. Der Kalkansatz steigt von 0,06 auf $0,20 \mathrm{~g}$ pro Tag: der Organismus tritt also in das Stadium der Regeneration.

Die Nachperiode, deren Daner allerdings zu kurz ist, um eine sichtliche Wendung hervorzurufen, macht sich im Versuch durch eine Mehrausscheidung geltend. Der unter dem Einfluss des Phosphors gewonnene Kalk verlässt den Körper wieder. In der kurzen Zeit von 11 Tagen ist kein dauernder Ansatz erfolgt. Man kann sich wohl vorstellen, der zurückgehaltene Kalk sei nur locker gebunden worden und gelange darum nach Aussetzen des die Regeneration bewirkenden Principes in erster Linie und besonders leicht wieder zur Ausscheidung;

Tabelle I. Nahrungsmittel.

$\mathrm{P}_{2} \mathrm{O}_{5}$ und $\mathrm{CaO}-G e h a l t$ der frischen Substanz in Procenten.

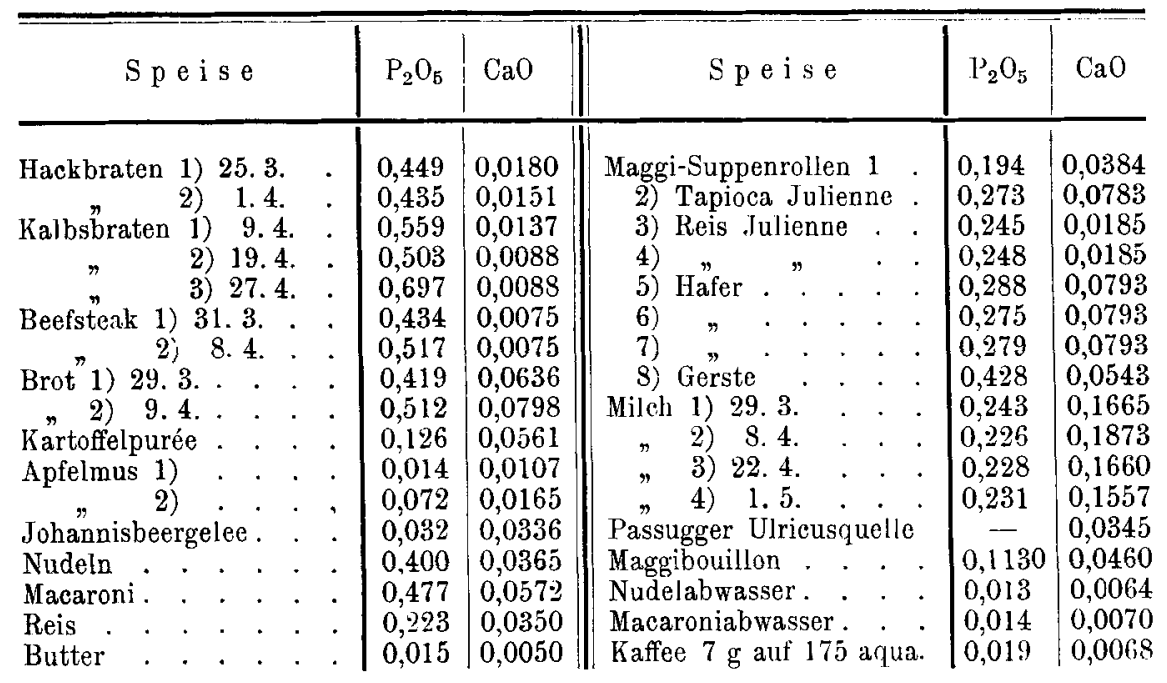

\begin{tabular}{|c|c|c|c|}
\hline & & $\mathrm{P}_{2} \mathrm{O}_{5}$ & $\mathrm{CaO}$ \\
\hline $\begin{array}{l}\text { Kohlekapseln, } \\
\text { Carminkapseln } \\
\text { 'Thyrenideatahl. }\end{array}$ & $\begin{array}{cc}\text { per } & \text { Stück } \\
. & . \\
. & .\end{array}$ & $0,006 \mathrm{~g}$ & 0.0215 \\
\hline
\end{tabular}


Tabelle II. Gasser.

$\mathrm{P}_{2} \mathrm{O}_{5}$ und $\mathrm{CaO}$ in den Ausscheidungen.

\begin{tabular}{|c|c|c|c|c|c|c|c|}
\hline \multirow{2}{*}{ Datum } & \multicolumn{4}{|c|}{$\mathrm{H}$ a $\mathrm{rn}$} & \multicolumn{3}{|c|}{$K \circ t h$} \\
\hline & Menge & $\begin{array}{l}\text { Spez. } \\
\text { Gew. }\end{array}$ & $\mathrm{P}_{2} \mathrm{O}_{5}$ & $\mathrm{CaO}$ & Menge & $\mathrm{P}_{2} \mathrm{O}_{5}$ & $\mathrm{CaO}$ \\
\hline
\end{tabular}

I. Vorperiod e.

\begin{tabular}{|c|c|c|c|c|c|c|c|}
\hline 29. März & 1790 & 1015 & 1,6890 & 0,1969 & 30,45 & 2,1847 & 2,1126 \\
\hline 30. & 1480 & 1014 & 1,1470 & 0,1302 & 30,45 & 2,1847 & 2,1126 \\
\hline $31 . \quad "$ & 1450 & 1015 & 1,6783 & 0,1450 & 24,05 & 1,8123 & 1,7792 \\
\hline 1. April & 1860 & 1013 & 1,4833 & 0,1525 & 23,96 & 1,6508 & 1,6819 \\
\hline 2. & 1470 & 1016 & 1,8411 & 0,1528 & 23,96 & 1,6508 & 1,6819 \\
\hline 3. & 1610 & 1014 & 1,5617 & 0,1392 & 23,96 & 1,6508 & 1,6819 \\
\hline 4. & 1340 & 1015 & 1,6314 & 0,1259 & 23.96 & 1,6508 & 1,6819 \\
\hline 5. & 1920 & 1014 & 1,8432 & 0,1862 & 23,96 & 1,6508 & 1,6819 \\
\hline 6. & 1730 & 1014 & 1,5007 & 0,1418 & 23,96 & 1,6508 & 1,6819 \\
\hline 7. " & 1880 & 1014 & 1,5181 & 0,1092 & 26,60 & 1,9777 & 1,8859 \\
\hline
\end{tabular}

II. Phos phor periode.

\begin{tabular}{rr|r|r|r|r|r|r|r} 
8. April & 1670 & 1012 & 1,4819 & 0,1553 & 29,00 & 2,1721 & 2,1953 \\
9. & $"$ & 2115 & 1011 & 1,5883 & 0,1731 & 17,70 & 1,2319 & 1,1841 \\
10. & $"$ & 1980 & 1013 & 1,8067 & 0,1235 & 31,25 & 2,1778 & 2,1468 \\
11. & $"$ & 1110 & 1017 & 1,1821 & 0,1076 & 22,80 & 1,5417 & 1,4683 \\
12. & $"$ & 2050 & 1013 & 1,7117 & 0,1476 & 24,15 & 1,7653 & 1,7338 \\
13. & $"$ & 1220 & 1018 & 1,5341 & 0,1329 & 17,20 & 1,2126 & 1,2642 \\
14. & $"$ & 1810 & 1015 & 1,6742 & 0,1556 & 17,20 & 1,2126 & 1,2642 \\
15. & & 1830 & 1014 & 1,4548 & 0,1720 & 27,72 & 2,0572 & 2,0235 \\
16. & $"$ & 1600 & 1013 & 1,4240 & 0,1312 & 27,72 & 2,0572 & 2,0235 \\
17. & & 1800 & 1014 & 1,3680 & 0,1503 & 22,15 & 1,6989 & 1,6789 \\
18. & & 1990 & 1013 & 1,4527 & 0,1661 & 22,15 & 1,6989 & 1,6789
\end{tabular}

III. Nach periode.

\begin{tabular}{ll|l|l|l|l|l|l|l} 
19. April & 1940 & 1012 & 1,4207 & 0,1465 & 22,60 & 1,7808 & 1,7582 \\
20. & $"$ & 1590 & 1017 & 1,1130 & 0,1669 & 22,60 & 1,7808 & 1,7582 \\
21. & $"$ & 1530 & 1017 & 1,1934 & 0,1790 & 18,90 & 1,6486 & 1,6159 \\
22. & $"$ & 1910 & 1013 & 1,2988 & 0,1776 & 18,90 & 1,6486 & 1,6159 \\
$\mathbf{2 3 .}$ & $\#$ & 2010 & 1010 & 1,1180 & 0,1548 & 24,75 & 2,1586 & 2,1606 \\
24. & & 1060 & 1019 & 1,3409 & 0,1420 & 24,75 & 2,1586 & 2,1606 \\
25. & $"$ & 1920 & 1012 & 1,2145 & 0,1728 & 22,50 & 1,8663 & 1,3607 \\
26. & $"$ & 1780 & 1013 & 1,4017 & 0,1548 & 22,50 & 1,8663 & 1,8607 \\
$\mathbf{2 7 .}$ & $"$ & 1420 & 1016 & 1,2886 & 0,1136 & 24,70 & 1,6853 & 1,7784 \\
28. & & 1460 & 1014 & 1,3541 & 0,1225 & 24,70 & 1,6853 & 1,7784
\end{tabular}

IV. Thy reoidea periode.

\begin{tabular}{|c|c|c|c|c|c|c|c|}
\hline 29. April & 1630 & 1014 & 1,4873 & 0,1222 & 19,55 & 1,5274 & 1,5190 \\
\hline 30. & 1750 & 1012 & 1,6537 & 0,1531 & 19,55 & 1,5274 & 1,5190 \\
\hline 1. Mai & 1470 & 1017 & 1,5398 & 0,1198 & 26,05 & 1,8000 & 1,8980 \\
\hline 2. & 1700 & 1014 & 1,6830 & 0,1810 & 26,05 & 1,8000 & 1,8980 \\
\hline 3. & 1715 & 1014 & 1,6249 & 0,1286 & 22,85 & 1,8965 & 1,7806 \\
\hline 4. & 1680 & 1012 & 1,1953 & 0,1134 & 22,85 & 1,8965 & 1,7806 \\
\hline 5. & 1420 & 1015 & 1,2460 & 0.1036 & 93,90 & 1,9048 & 1.8044 \\
\hline 6. & 1820 & 1010 & 1.3240 & 0.1574 & 23.90 & 1,9048 & 1.8044 \\
\hline
\end{tabular}


Tabelle III. Gasser.

Tagesbilanz.

\begin{tabular}{|c|c|c|c|c|c|c|}
\hline \multirow{2}{*}{ Datum } & \multicolumn{3}{|c|}{$P_{2} O_{5}$} & \multicolumn{3}{|c|}{$\mathrm{CaO}$} \\
\hline & Einnahme & Ausgabe & Differenz & Einnahme & Ausgabe & Differenz \\
\hline
\end{tabular}

I. Vorperiode.

\begin{tabular}{|c|c|c|c|c|c|c|}
\hline 29. März & 3,7890 & 3,8737 & $-0,0847$ & 2,0915 & 2,3095 & $-0,2180$ \\
\hline 30. " & 3,7110 & 3,3317 & $+0,3793$ & 2,2158 & 2,2428 & $-0,0270$ \\
\hline 31. & 3,3951 & 3,4906 & $-0,0955$ & 2,0104 & 1,9242 & $+0,0862$ \\
\hline 1. April & 3,3571 & 3,1341 & $+0,2230$ & 1,9914 & 1,8344 & $+0,1570$ \\
\hline $2, \quad$, & 3,3789 & 2,4919 & $-0,1130$ & 1,9534 & 1,8347 & $+0,1187$ \\
\hline 3. & 3,3546 & 3,2125 & $+0,1421$ & 1,9847 & 1,8211 & $+0,1636$ \\
\hline 4. & 3,4189 & 3,2822 & $+0,1367$ & 1,9717 & 1,8078 & $+0,1639$ \\
\hline 5. & 3,6797 & 3,4940 & $+0,1857$ & 2,0168 & 1,8681 & $+0,1487$ \\
\hline 6. & 3,1239 & 3,15 & $-0,0276$ & 1,6982 & 1,8237 & $-0,1255$ \\
\hline 7. & 4,1045 & 3,4758 & +0.6287 & 2,1898 & 2,0551 & $+0,1347$ \\
\hline &, 3127 & 3,9380 & + 1,3747 & 0,1237 & 19,5214 & $+0,6023$ \\
\hline
\end{tabular}

II. Phosphorperiode.

\begin{tabular}{|c|c|c|c|c|c|c|}
\hline 8. April & 3,3287 & 3,6540 & $-0,3253$ & 2,0536 & 2,3506 & $-0,2970$ \\
\hline 9. " & 3,7281 & 2,8202 & $+0,9079$ & 2,1673 & 1,3572 & $+0,8101$ \\
\hline 10. & 3,6246 & 3,9845 & $-0,3599$ & 2,1948 & 2,2703 & $-0,0755$ \\
\hline 11. & 3,7504 & 2,7238 & $+1,0266$ & 1,9895 & 1,5759 & $+0,4136$ \\
\hline 12. & 3,2924 & 3,4770 & $-0,1846$ & 2,0226 & 1,8864 & $+0,1362$ \\
\hline 13. & 3,5874 & 2,7467 & $+0,8407$ & 2,0073 & 1,3971 & $+0,6102$ \\
\hline 14. & 3,6021 & 2,8868 & $+0,7153$ & 2,0073 & 1,4198 & $+0,5878$ \\
\hline 15. & 3,7246 & 3,5120 & $+0,2126$ & 2,0405 & 2,1955 & $-0,1550$ \\
\hline 16. & 3,1301 & 3,4812 & $-0,3511$ & 1,9601 & 2,1547 & $-0,1946$ \\
\hline 17. & 3,4821 & 3,0669 & $+0,4152$ & 2,1372 & 1,8292 & $+0,3080$ \\
\hline 18. & 3,1764 & 3,1516 & +0.0248 & 1,9792 & 1.8450 & $+0,1342$ \\
\hline Summa & 38,4269 & 35,5047 & $+2,9222$ & 22,5594 & 20,2817 & $+2,2777$ \\
\hline
\end{tabular}

III. $\mathrm{N}$ a c h periode.

\begin{tabular}{|c|c|c|c|c|c|c|}
\hline 19. April & 3,4908 & 3,2015 & †. 0,2893 & 2,0160 & 1,9037 & $+0,1123$ \\
\hline 20. & 3,5031 & 2,8938 & $+0,6093$ & 2,0229 & 1,9251 & $+0,0978$ \\
\hline 21. & 3,5398 & 2,8420 & $\begin{array}{r}+0,6978 \\
\end{array}$ & 2,1561 & 1,7949 & 0,3612 \\
\hline 22. & 3,2011 & 2,9474 & $\begin{array}{l}+0,2537 \\
\end{array}$ & 1,8492 & 1,793 & $+0,0557$ \\
\hline 23. & 3,5620 & 3,2766 & $\begin{array}{l}+0,2854 \\
\end{array}$ & 2,2602 & $2, \mathrm{~B} 1$ & $-0,0552$ \\
\hline 24. & 3,2674 & 3,4995 & $-0,2321$ & 1,8021 & 2,302 & $-0,5005$ \\
\hline 25 . & 3,7140 & 3,0808 & $+0,6332$ & 2,1354 & 2,0335 & $+0,1019$ \\
\hline 26. & 3,0138 & 3,2680 & $-0,2542$ & 1,3331 & 2,0155 & $\therefore 0,6824$ \\
\hline 27. & 3,7797 & 2,9739 & $+0,8058$ & 1,9782 & 1,8920 & $+0,0862$ \\
\hline 28. & 3,6484 & 3,0394 & $\begin{array}{r}\quad 0,6090 \\
\end{array}$ & 1,8003 & 1,9009 & $-0,1006$ \\
\hline Summ: & 34,7201 & 31,0229 & $+3,6972$ & 19,3535 & 19,8771 & 0,5236 \\
\hline
\end{tabular}

IV. Thyreoideaperiode.

\begin{tabular}{|c|c|c|c|c|c|c|}
\hline 29. April & 3,9008 & 3,0147 & $+0,8861$ & 1,8641 & 1,6412 & $+0,2229$ \\
\hline 30. & 3,4071 & 3,1811 & $+0,2260$ & 1,9489 & 1,6721 & $+0,2768$ \\
\hline 1. Mai & 3,8420 & 3,3398 & 0,5022 & 1,8116 & 2,0178 & 0,2062 \\
\hline 2. $"$ & 3,7900 & 3,4830 & $+0,3070$ & 1,8887 & 2,0790 & 0,1903 \\
\hline 3. & 3,8657 & 3,5210 & $+0,3447$ & 1.9272 & 1,9092 & $+0,0180$ \\
\hline 4. & 3,8111 & 3,0918 & $+0,7193$ & 1,8485 & 1,8940 & $-0,0455$ \\
\hline 5. " & 4,0380 & $3,150 \mathrm{~s}$ & $+0,8872$ & 2,0206 & 1,9080 & $+0,1126$ \\
\hline 6. & 3,5300 & 3,2288 & $+0,3012$ & 1,8761 & 1,9618 & 0,0857 \\
\hline Summa & 30,1847 & 26,0110 & 4.1737 & 15,1857 & 15,0831 & +0.1026 \\
\hline
\end{tabular}


Tabelle IV. Gasser.

\begin{tabular}{|c|c|c|c|c|c|}
\hline & & \multicolumn{2}{|c|}{$\mathrm{P}_{2} \mathrm{O}_{5}$-Bilanz } & \multicolumn{2}{|c|}{$\mathrm{CaO}-\mathrm{Bilan} \%$} \\
\hline & & $\begin{array}{c}\text { Gesammt } \\
\mathrm{g}\end{array}$ & $\begin{array}{c}\text { pro Tag } \\
\mathrm{g}\end{array}$ & $\begin{array}{l}\text { Gesammt } \\
\mathrm{g}\end{array}$ & $\begin{array}{c}\text { pro Tag } \\
\mathrm{g}\end{array}$ \\
\hline \multirow[t]{2}{*}{$\begin{array}{l}\text { Vorperiode } \\
10 \text { Tage }\end{array}$} & $\begin{array}{l}\text { Einfuhr } \\
\text { Ausfuhr }\end{array}$ & $\begin{array}{l}35,3127 \\
33,9380\end{array}$ & $\begin{array}{l}3,53127 \\
3,39380 \\
\end{array}$ & $\begin{array}{l}20,1237 \\
19,5214 \\
\end{array}$ & $\begin{array}{l}2,0122 \\
1,9521 \\
\end{array}$ \\
\hline & & \multicolumn{2}{|c|}{ Retention } & \multicolumn{2}{|c|}{ Retention } \\
\hline $\begin{array}{c}\text { Phosphorperiode } \\
11 \text { Tage }\end{array}$ & $\begin{array}{l}\text { Einfuhr } \\
\text { Ausfuhr }\end{array}$ & $\begin{array}{l}38,4269 \\
35,5047\end{array}$ & $\begin{array}{l}3,4933 \\
\mathbf{3}, 2277\end{array}$ & $\begin{array}{l}22,5594 \\
20,2817\end{array}$ & $\begin{array}{l}2,0508 \\
1,8438 \\
\end{array}$ \\
\hline \multicolumn{2}{|l|}{ Phosphor 0,001 p. die. } & \multicolumn{2}{|c|}{ Retention } & \multicolumn{2}{|c|}{ Retention } \\
\hline \multirow[t]{2}{*}{$\begin{array}{l}\text { Nachperiode } \\
10 \text { Tage }\end{array}$} & $\begin{array}{l}\text { Einfuhr } \\
\text { Ausfuhr }\end{array}$ & $\begin{array}{l}34,7201 \\
31,0229 \\
\end{array}$ & $\begin{array}{l}3,47201 \\
3,10229 \\
\end{array}$ & $\begin{array}{l}19,3535 \\
19,8771 \\
\end{array}$ & $\begin{array}{r}1,9353 \\
1,9877 \\
\end{array}$ \\
\hline & & Retention & $\begin{array}{l}0,36972 \\
\text { tion }\end{array}$ & $\begin{array}{l}-0,5236 \\
\text { Mehrauss }\end{array}$ & $\begin{array}{l}-0,05236 \\
\text { heidung! }\end{array}$ \\
\hline $\begin{array}{c}\text { Thyreoideaperiode } \\
\text { 8 Tage }\end{array}$ & $\begin{array}{l}\text { Einfuhr } \\
\text { Ausfuhr }\end{array}$ & $\begin{array}{l}30,1847 \\
26,0110 \\
\end{array}$ & $\begin{array}{l}3,7731 \\
3,2514 \\
\end{array}$ & $\begin{array}{l}15,1857 \\
15,0831 \\
\end{array}$ & $\begin{array}{l}1,8982 \\
1,8854 \\
\end{array}$ \\
\hline 3 Tabl. à 0,3 & & $\begin{array}{r}+4,1737 \\
\text { Rete }\end{array}$ & $\begin{array}{l}0,5217 \\
\text { tion }\end{array}$ & $\begin{array}{r}+0,1026 \\
\text { Rete }\end{array}$ & $\begin{array}{l}+0,0128 \\
\text { tion }\end{array}$ \\
\hline
\end{tabular}

sobald dies geschehen ist, während der Thyreoideaperiode, kehrt der Organismus, was das Calcium anbetrifft, wieder in den Zustand der Vorperiode mit geringem Kalkansatz zurück. Die specifische Wirkung des Phosphors bei Osteomalacie, denn eine solche anzunehmen, rechtfertigt neben den bekannten klinischen Erfahrungen auch dieser Versuch, ist demgemäss nicht so aufzufassen, dass mit einem Schlage ein dauernder Umschwung im Kalkstoffwechsel auftrete. Die Regeneration steigt, aber sie fällt auch sofort mit Einführen und Aussetzen des Phosphors. Wir sehen daraus, dass die Phosphortherapie bei Osteomalacie nur dann Erfolg hat, wenn sie durch lange Zeit continuirlich angewandt, davernd die Halisteresis unterdrückt.

Berechnet man, was der Organismus unserer ersten Patientin während des ganzen Versuches an Kalk gewonnen hat, so ergiebt sich durch Addition der zurückgehaltenen Mengen während der 29 Tage der Vorphosphor- und Thyreoideaperiode, abzüglich des Verlustes in der Nachperiode eine Gesammtretention von $1,8567 \mathrm{~g}$ CaO. Nimmt man nun an, die Tendenz des Körpers, Kalk anzusetzen, wie sie sich in der Vorperiode durch eine Retention von $0,0602 \mathrm{~g} \mathrm{CaO}$ pro Tag kundgiebt, hätte während der ganzen Versuchszeit angehalten, so ergäbe sich eine voraussichtliche Kalkretention von $29 \times 0,0602=1,7458 \mathrm{~g}$. In Wirklichkeit sind $1,8567 \mathrm{~g}$ gewonnen worden.

Diese beiden Zahlen decken sich beinahe vollkommen und beweisen eben, dass nur eine durch Monate oder Jahre fortgesetzte Phosphortherapie den früheren Kalkverlust wieder einbringen kann. 
Die Phosphorsäurebilanz zeigt in der

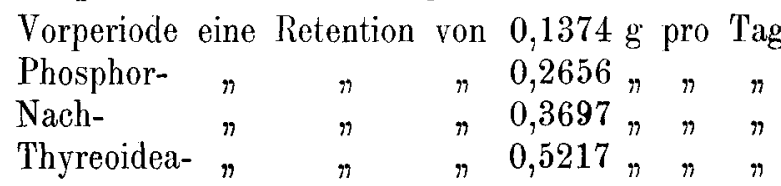

Im Gegensatz zu den Schwankungen des Kalkes ergiebt sich eine regelmässig rasch zunehmende $\mathrm{P}_{2} \mathrm{O}_{5}$ Aufspeicherung, welche anscheinend von der Phosphortherapie nicht beeinflusst wird. Zweifellos steht dieses Verhalten mit der Zunahme des Körpergewichtes in engem Zusammenhang. Die $\mathrm{P}_{2} \mathrm{O}_{5}$-Retention beträgt während des ganzen Versuches $12,16 \mathrm{~g}$. Das Körpergewicht steigt um $3,7 \mathrm{~kg}$. Berechnen wir diesen Gewinn von 12,16 g $\mathrm{P}_{2} \mathrm{O}_{5}$ auf Musculatur, so finden wir unter der Annahme, das Muskelfleisch enthalte 3,4 prom. Phosphorsäure $\left.{ }^{1}\right) 3,5 \mathrm{~kg}$. Bei unserer Patientin beträgt die Gewichtszunahme $3,7 \mathrm{~kg}$. Die Uebereinstimmung dieser Zahlen liesse sich verwerthen für die Annahme, die $\mathrm{P}_{2} \mathrm{O}_{5}$ möchte in der gekräftigten Musculatur Verwendung gefunden haben; eine exacte Kenntniss über die Vertheilung der zurückgehaltenen $\mathrm{P}_{2} \mathrm{O}_{5}$ könnte aber nur eine Analyse des gesammten Stoffumsatzes garantiren.

Für den 2. Versuch. Bei Frau Gehrig ist die Tabellenordnung die Gleiche wie bei Frau Gasser.

Tabelle V. Menge, specifisches Gewicht, $\mathrm{P}_{2} \mathrm{O}_{5}-$ und $\mathrm{CaO}$-Gehalt im Harn und Koth der einzelnen Tage.

Tabelle VI. Tagesbilanz.

Tabelle VII. Calcium und Phosphorbilanz der vier Perioden.

Tabelle VIII giebt eine graphische Darstellung der Resultate des Stoffwechselversuches bei beiden Fällen.

\section{Calciumbilanz}

in der Vorperiode Mehrausscheidung von $1,9550 \mathrm{~g}=0,2443 \mathrm{~g}$ pro Tag

" "Phosphor- Retention "0,3417 " $=0,0284 "$ " "

$" n$ Nach- Mehrausscheidung " $0,0246,=0,0035 n " n "$

" "Thyreoidea- " " $1,2388 "=0,1548, " n$

Die Vorperiode setzt sofort nach der Spitalaufnahme ein, sie zeigt uns die Kranke in einem noch wenig beeinflussten Zustande, im floriden Kalkabbau. Bedenken wir, dass die Halisteresis bereits seit Jahren am Skelett zehrt, so muss die Mehrausscheidung von täglich $0,24 \mathrm{~g} \mathrm{CaO}$ als eine hohe bezeichnet werden. Nun wird Phosphor gegeben. Mit einem Schlage ändert sich die Bilanz, der grosse Kalkverlust sistirt; es ergiebt sich sogar eine kleine Retention. Die Krankheit steht still, die Ausgleichung des Verlustes beginnt. Während der folgenden sieben Tage wird der Phosphor wieder ausgesetzt. Der Einfluss der Entziehung wird in diesem Falle nicht klar illustrirt. Ls dürften wohl die ersten Tage noch unter der Nachwirkung des Medi-

1) Hamarsten, Lehrb. d. phys. Chemie. 1899. S. 362. 
T a b e $1 \mathrm{l}$ e V. Gehrig.

$\mathrm{P}_{2} \mathrm{O}_{5}$ und $\mathrm{CaO}$ in den Ausscheidungen.

\begin{tabular}{l|l|l|l|l|l|l|l}
\hline \multirow{2}{*}{ Datum } & \multicolumn{3}{|c|}{$\mathrm{Harn}$} & \multicolumn{3}{c}{$\mathrm{K}$ ot h } \\
\cline { 2 - 5 } & Menge & $\begin{array}{c}\text { Spez. } \\
\text { Gew. }\end{array}$ & $\mathrm{P}_{2} \mathrm{O}_{5}$ & $\mathrm{CaO}$ & Menge & $\mathrm{O}_{2} \mathrm{O}_{5}$ & $\mathrm{CaO}$ \\
\hline
\end{tabular}

I. Vorperiode.

\begin{tabular}{|c|c|c|c|c|c|c|c|}
\hline 1. April & 1650 & 1013 & 0,9545 & 0,1419 & 22,1 & 1,5205 & 1,4019 \\
\hline 2. & 930 & 1013 & 0,5533 & 0,1748 & 22,1 & 1,5205 & 1,4019 \\
\hline 3. & 1580 & 1014 & 1,5035 & 0,1706 & 22,1 & 1,5205 & 1,4019 \\
\hline 4. & 1250 & 1014 & 1,1875 & 0,1225 & 22,1 & 1,5205 & 1,4019 \\
\hline 5. & 1505 & 1014 & 1,0950 & 0,1455 & 22,1 & 1,5205 & 1,4019 \\
\hline 6. & 1150 & 1017 & 0,9671 & 0,0989 & 22,1 & 1,5205 & 1,4019 \\
\hline 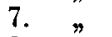 & 1170 & 1018 & 1,0647 & 0,1579 & 36,0 & 2,2237 & 1,8087 \\
\hline 8 & 1430 & 1014 & 1,3513 & 0,1644 & 24,2 & 1,3012 & 1,3374 \\
\hline
\end{tabular}

II. Phosphorperiode.

\begin{tabular}{rl|r|r|r|l|l|l|l}
9. & April & 1450 & 1014 & Verlust & Verlust & 24,2 & 1,3012 & 1,3374 \\
10. & $"$ & 1290 & 1014 & 1,2900 & 0,1367 & 24,2 & 1,3012 & 1,3374 \\
11. & $\#$ & 1850 & 1014 & 1,8870 & 0,1924 & 31,9 & 1,6977 & 1,3692 \\
12. & $\#$ & 1320 & 1015 & 1,2144 & 0,1597 & 17,6 & 1,1157 & 1,2165 \\
13. & $"$ & 1200 & 1016 & 1,3230 & 0,1404 & 14,7 & 0,9339 & 0,9034 \\
14. & $"$ & 1180 & 1018 & 1,3015 & 0,1463 & 14,7 & 0,9339 & 0,9034 \\
15. & $\#$ & 1070 & 1016 & 1,0165 & 0,1722 & 22,1 & 1,5866 & 1,5503 \\
16. & $"$ & 1040 & 1016 & 0,9880 & 0,1518 & 22,1 & 1,5866 & 1,5503 \\
17. & $"$ & 1020 & 1019 & 1,1577 & 0,1020 & 21,2 & 1,5055 & 1,4598 \\
18. & $"$ & 900 & 1022 & 1,2550 & 0,1494 & 21,2 & 1,5055 & 1,4598 \\
19. & $"$ & 1140 & 1014 & 1,5330 & 0,1117 & 22,0 & 1,6227 & 1,2625 \\
20. & $"$ & 760 & 1022 & Verlust & Verlust & 22,0 & 1,6227 & 1,2625
\end{tabular}

III. Nachperiode.

\begin{tabular}{|c|c|c|c|c|c|c|c|}
\hline 21. April & 840 & 1019 & 1,0542 & 0,0848 & 22,8 & 1,5579 & 1,2966 \\
\hline 22. & 960 & 1020 & Verlust & Verlust & 22,8 & 1,5579 & 1,2966 \\
\hline 3. & 910 & 1020 & 0,9132 & 0,1374 & 26,7 & 1,4416 & 1,0637 \\
\hline 4. & 770 & 1020 & 1,0452 & 0,1009 & 26,7 & 1,4416 & 1,0637 \\
\hline 5. & 1145 & 1016 & 1,0604 & 0,1488 & 22,3 & 1,6 & 1,3535 \\
\hline 6. & 1240 & 1015 & 1,2493 & & 22,3 & 1,6586 & 1,3535 \\
\hline 7. & 850 & 1020 & 1,0259 & 0,1127 & 22,6 & 1,4589 & 1,0683 \\
\hline
\end{tabular}

IV. Thyreoideaperiode.

\begin{tabular}{cc|r|c|c|c|c|c|c} 
28. April & 1068 & 1019 & 1,3675 & 0,1091 & 22,6 & 1,4589 & 1,0683 \\
29. & 970 & 1014 & 0,9816 & 0,1756 & 28,0 & 1,6568 & 1,2732 \\
30. & M" & 690 & 1018 & 0,6590 & 0,0593 & 28,0 & 1,6568 & 1,2732 \\
1. Mai & 1280 & 1014 & 0,7654 & 0,0922 & 28,4 & 1,5935 & 1,4419 \\
2. & 920 & 1019 & 0,8326 & 0,1637 & 28,4 & 1,5935 & 1,4419 \\
3. & & 1060 & 1016 & 1,2270 & 0,1378 & 31,4 & 2,2143 & 1,6524 \\
4. " & 860 & 1013 & 0,6845 & 0,2400 & 31,4 & 2,2143 & 1,6524 \\
5. & & 870 & 1018 & 0,9765 & 0,2227 & 22,1 & 1,2555 & 1,1546
\end{tabular}


Tabelle VI. Gehrig.

Tagesbilanz.

\begin{tabular}{|c|c|c|c|c|c|c|}
\hline \multirow{2}{*}{ Datum } & \multicolumn{3}{|c|}{$\mathrm{P}_{2} \mathrm{O}_{5}$} & \multicolumn{3}{|c|}{$\mathrm{CaO}$} \\
\hline & Einnahme & Ausgabe & Differenz & Einnahme & Ausgabe & Differenz \\
\hline
\end{tabular}

I. Vorperiode.

\begin{tabular}{|c|c|c|c|c|c|c|}
\hline 1. April & 2,8100 & 2,4750 & $+0,3350$ & 1,3629 & 1,5438 & $-0,1809$ \\
\hline$\eta$ & 2,4453 & 2,0738 & $\begin{array}{l}+0,3715 \\
\end{array}$ & 1,2774 & 1,5767 & $-0,2993$ \\
\hline 3. & 2,5683 & 3,0240 & $-0,4557$ & 1,3353 & 1,5725 & $-0,2372$ \\
\hline 4. & 2,7670 & 2,7080 & $+0,0590$ & $\mathbf{1 , 3 3 8 8}$ & 1,5244 & - 0,1856 \\
\hline , & 3,1943 & 2,6155 & $+0,5788$ & 1,4172 & 1,5474 & $-0,1302$ \\
\hline 6. & 2,7114 & 2,4876 & 0,2238 & 1,2667 & 1,5008 & - 0,2341 \\
\hline " & 2,7509 & 3,2884 & $-0,5375$ & 1,2519 & 1,9666 & $-0,7147$ \\
\hline , & 2,9006 & 2,6525 & $+0,2481$ & 1,5288 & 1,5018 & $\begin{array}{r}+0,0270 \\
\end{array}$ \\
\hline $\mathrm{mm}$ & 22,1478 & 21,3248 & $+0,8230$ & 10,7790 & 12,7340 & $-1,9950$ \\
\hline
\end{tabular}

II. Phosphorperiode.

\begin{tabular}{|c|c|c|c|c|c|c|}
\hline 9. April & 2,8858 & 1) 2,6218 & {$[1)+0,2640$} & 1,4631 & 1) 1,4879 & 1) $-0,0248$ \\
\hline 10. & 2,7438 & 2,5912 & $+0,1526$ & 1,4671 & 1,4741 & $-0,0070$ \\
\hline 11. & 2,8170 & 3,5847 & $-0,7677$ & 1,4533 & 1,5616 & $-0,1083$ \\
\hline 12. & 2,3774 & 2,3301 & $+0,0473$ & 1,4329 & 1,3762 & $+0,0567$ \\
\hline 13. & 2,4410 & 2,2569 & $+0,18$ & 1,3827 & 1,0438 & 0,3389 \\
\hline 14. & 2,8414 & 2,2354 & $+0,6060$ & 1,4420 & 1,0497 & $+0,3923$ \\
\hline 15. & 2,4325 & 2,6031 & $-0,1706$ & 1,4059 & 1,7225 & $-0,3166$ \\
\hline 16. & 2,3958 & 2,5746 & $-0,1788$ & 1,3907 & 1,70 & $-0,3114$ \\
\hline 17. & 2,5726 & 2,6632 & $-0,0906$ & 1,4425 & 1,5618 & $-0,1193$ \\
\hline 18. & 2,5341 & 2,7605 & $-0,2264$ & 1,4377 & 1,60 & $-0,1715$ \\
\hline 19. & 2,999 & 3,1557 & $-0,1567$ & 1,9525 & 1,3742 & $+0,5783$ \\
\hline 20. & 2,4369 & 1) 2,9163 & 1) $-0,4794$ & 1,3951 & (1) 1,3607 & 1) $+0,0344$ \\
\hline & 4773 & 32,2935 & $-0,8162$ & 17,6655 & 17,3238 & $+0,3417$ \\
\hline
\end{tabular}

III. $\mathrm{N}$ a c h periode.

\begin{tabular}{|c|c|c|c|c|c|c|}
\hline 21. April & 2,5462 & 2,6121 & $-0,0659$ & 1,4384 & 1,3814 & $+0,0570$ \\
\hline 22. & 2,2917 & 1) 2,5416 & 1) $-0,2499$ & 1,3137 & 1) 1,4077 & 1) $-0,0940$ \\
\hline 3. & 2,6507 & 2,3548 & $+0,2959$ & 1,2959 & 1,2011 & $+0,0948$ \\
\hline 24. & 2,5186 & 2,4868 & $+0,0318$ & 1,2430 & 1,1646 & $+0,0784$ \\
\hline 55. & 2,7861 & 2,7190 & $+0,0671$ & 1,4796 & 1,50 & $-0,0227$ \\
\hline 26. & 2,8228 & 2,9079 & $-0,0851$ & 1,2852 & 1,5023 & $-0,2171$ \\
\hline 27. & 2,7727 & 2,4848 & $\begin{array}{r}+0,2879 \\
\end{array}$ & 1,2600 & 1,1810 & $+0,0790$ \\
\hline Summ & 18,3888 & 18,1070 & $+0,2818$ & 9,3158 & 9,3404 & $-0,0246$ \\
\hline
\end{tabular}

IV. Thyreoideaperiode.

\begin{tabular}{|c|c|c|c|c|c|c|}
\hline 28. April & 2,6150 & 2,8264 & $-0,2114$ & 1,2834 & 1,1774 & $+0,1060$ \\
\hline 29. & 2,6499 & 2,6384 & $+0,0115$ & 1,2957 & 1,4488 & $-0,1531$ \\
\hline & 2,5946 & 2,3158 & $+0,2788$ & 1,3038 & 1,3325 & $-0,0287$ \\
\hline 1. Mai & 2,2261 & 2,3589 & $-0,1328$ & 1,2250 & 1,5341 & $-0,3091$ \\
\hline 2. & 3,0686 & 2,4261 & $+0,6425$ & 1,4352 & 1,6056 & $-0,1704$ \\
\hline 3. & 3,65599 & 3,4413 & $\begin{array}{r}+ \\
+\quad 0,2186\end{array}$ & 1,8274 & 1,7902 & $\begin{array}{r}+0,0372 \\
\end{array}$ \\
\hline 4. & 2,9751 & 2,8988 & $+0,0763$ & 1,2004 & 1,8924 & $-0,6920$ \\
\hline 5. & 3,0301 & 2,2320 & $\begin{array}{r}+ \\
+\quad 0,7981\end{array}$ & 1,3486 & 1,3773 & $-0,0287$ \\
\hline Summa & 22,8193 & 21,1377 & $+1,6816$ & 10,9195 & 12,1583 & $-1,2388$ \\
\hline
\end{tabular}

1) Für den Urinverlust ist der Durchschnittswerth aus der dem Versuchstage voransgehenden und nachfolgenden Tagesmenge eingesetzt. Vergl, S. 611. 
Tabell e VII. Gehrig.

\begin{tabular}{|c|c|c|c|c|c|}
\hline & & \multicolumn{2}{|c|}{$\mathrm{P}_{2} \mathrm{O}_{5}-$ Bilanz } & \multicolumn{2}{|c|}{$\mathrm{CaO}$-Bilanz } \\
\hline & & Total & pro Tag & Total & pro Tag \\
\hline \multirow[t]{2}{*}{$\begin{array}{l}\text { Vorperiode } \\
8 \text { Tage }\end{array}$} & $\begin{array}{l}\text { Einfuhr } \\
\text { A usfuhr }\end{array}$ & $\begin{array}{l}22,1478 \\
21,3248\end{array}$ & $\begin{array}{l}2,7684 \\
2,6656\end{array}$ & $\begin{array}{l}10,7790 \\
12,7340\end{array}$ & $\begin{array}{l}1,3474 \\
1,5917 \\
\end{array}$ \\
\hline & & Retention & $\begin{array}{l}+0,1028 \\
\text { tion }\end{array}$ & \multicolumn{2}{|c|}{ Mehrausgabe } \\
\hline \multirow[t]{2}{*}{$\begin{array}{c}\text { Phosphorperiode } \\
12 \text { Tage }\end{array}$} & $\begin{array}{l}\text { Einfuhr } \\
\text { Ausfuhr }\end{array}$ & $\begin{array}{l}31,4773 \\
32,2935 \\
\end{array}$ & $\begin{array}{l}2,6231 \\
2,6911\end{array}$ & $\begin{array}{l}17,6655 \\
17,3238\end{array}$ & $\begin{array}{l}1,4721 \\
1,4436 \\
\end{array}$ \\
\hline & & $\begin{array}{r}-0,8162 \\
\text { Mehra }\end{array}$ & $\begin{array}{l}-0,0680 \\
\text { sgabe }\end{array}$ & $\begin{array}{r}+0,3417 \\
\text { Rete }\end{array}$ & $\begin{array}{l}+0,0284 \\
\text { ation }\end{array}$ \\
\hline \multirow[t]{2}{*}{$\begin{array}{l}\text { Nachperiode } \\
7 \text { Tage }\end{array}$} & $\begin{array}{l}\text { Einfuhr } \\
\text { Ausfuhr }\end{array}$ & $\begin{array}{l}18,3888 \\
18,1070\end{array}$ & $\begin{array}{l}2,6269 \\
2,5869 \\
\end{array}$ & $\begin{array}{l}9,3158 \\
9,3404 \\
\end{array}$ & $\begin{array}{l}1,3308 \\
1,3343 \\
\end{array}$ \\
\hline & & $\begin{array}{r}+0,2818 \\
\text { Rete }\end{array}$ & $\begin{array}{l}+0,0402 \\
\text { tion }\end{array}$ & $\begin{array}{r}-0,0246 \\
\text { Mehra }\end{array}$ & $\begin{array}{l}-0,0035 \\
\text { usgabe }\end{array}$ \\
\hline \multirow[t]{2}{*}{$\begin{array}{c}\text { Thyreoideaperiode } \\
8 \text { Tage }\end{array}$} & $\begin{array}{l}\text { Einfuhr } \\
\text { Ausfuhr }\end{array}$ & $\begin{array}{l}22,8193 \\
21,1377\end{array}$ & $\begin{array}{l}2,8524 \\
2,6422 \\
\end{array}$ & $\begin{array}{l}10,9195 \\
12,1583\end{array}$ & $\begin{array}{l}1,3649 \\
1,5198 \\
\end{array}$ \\
\hline & & $\begin{array}{r}+1,6816 \\
\text { Rete }\end{array}$ & $\begin{array}{l}+0,2102 \\
\text { tion }\end{array}$ & $\begin{array}{r}-1,2388 \\
\text { Mehra }\end{array}$ & $\begin{array}{l}-0,1548 \\
\text { sqabe }\end{array}$ \\
\hline
\end{tabular}

camentes stehen; denn in der Tagesbilanz vom 21. bis 24. April treffen wir immer noch positive Werthe, erst vom 25. an setzt eine grössere Mehrausscheidung ein, welche den anfänglichen Gewinn der ganzen Nachperiode in Verlust umwandelt. In der vierten Periode schliesslich finden wir wiederum die ursprünglichen hohen negativen Differenzen. Der Process kehrt nach vorübergehender künstlicher Unterbrechung wieder in das Stadium des raschen Kalkabbaues zurück. Im Ganzen sind in 35 Tagen $2,87 \mathrm{~g}$ CaO dem Körper entzogen worden.

Einen gänzlich verschiedenen Verlauf nimmt die

Phosphorsäurebilanz

in der Vorperiode eine tägl. Retention von $0,1028 \mathrm{~g}$

" "Phosphor- " $"$ Mehrausscheidung " 0,0680 n

$"$ "Nach- " $"$ Retention " $0,0402 "$

" " Thyreoidea- " " " 0,2102 "

Die graphische Darstellung zeigt uns das directe Spiegelbild vom Calciumstoffwechsel. Neben einer Mehrausscheidung von Kalk eine $\mathrm{P}_{2} \mathrm{O}_{5}$-Retention in der Torperiode. Die Phosphortherapie bewirkt Kalkansatz; aber eine ebenso geringe $\mathrm{P}_{2} \mathrm{O}_{5}$-Mehrausscheidung. In der Nachperiode der wieder einsetzende Kalkverlust und eine wenig grössere Phosphoretention, welche in der Thyreoideaperiode, bei Abfall der Calciumeurve auf die ursprünglichen Werthe die anfängliche Retention noch übertrifft. Im Ganzen gewinnt der Organismus 3,7682 $\mathrm{g}$, aber das Körpergewicht bleibt constant.

Klinisch unterscheidet sich dieser zweite Fall von dem ersten durch eine viel hochgradigere Störung des Allgemeinbefindens, durch einen 

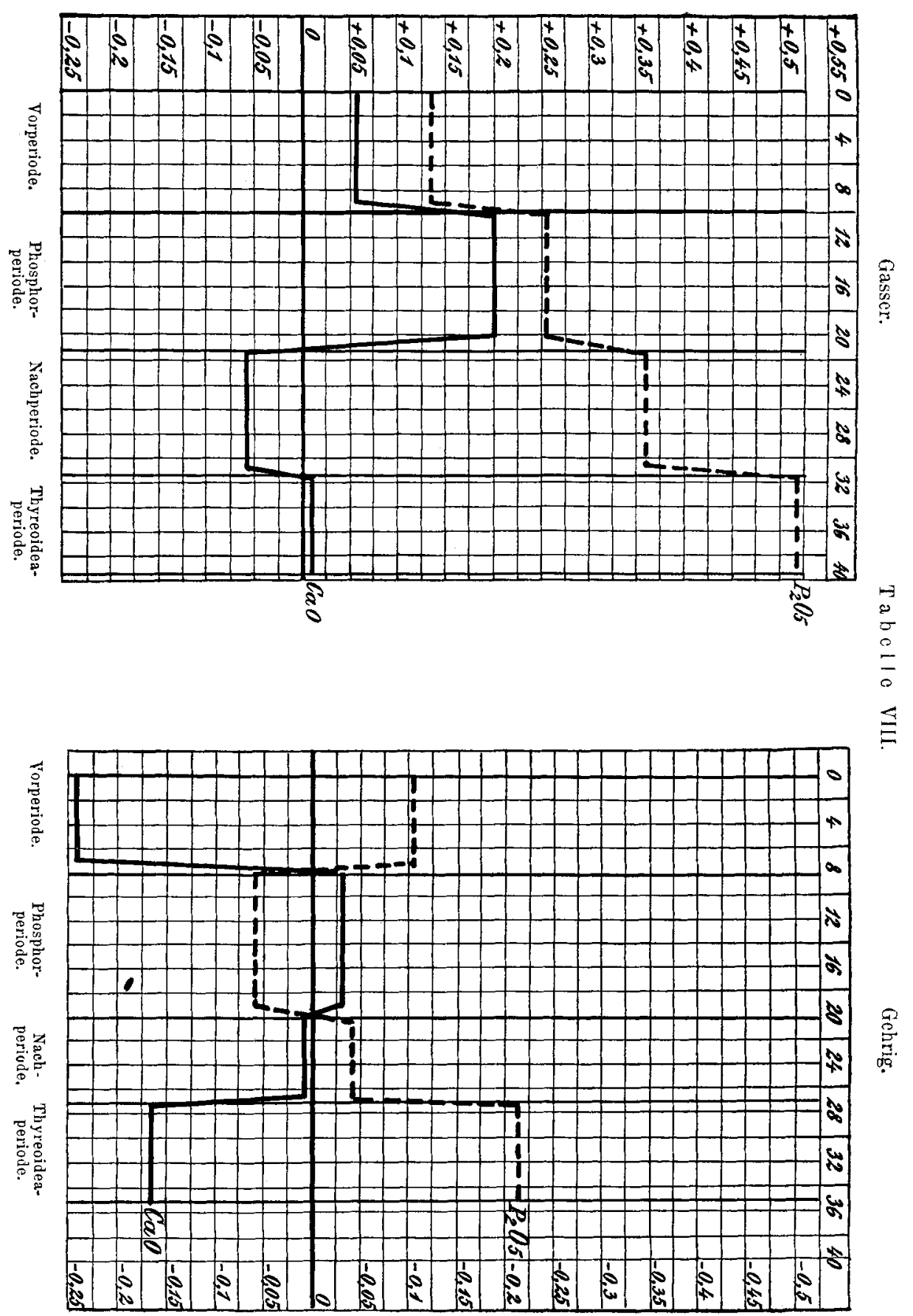
steten Fortschritt zum Schlimmeren. Die Phosphortherapie, die bei Frau Gasser sofortige Besserung der Beschwerden und eine baldige Wiederherstellung der Arbeitsfähigkeit erzielte, welche seither anhält, brachte der Anderen erst nach längerer Zeit Linderung der Schmerzen, erst nach Monaten konnte sie das Bett verlassen. $\mathrm{Zu}$ einer völligen Wiederherstellung ist es nie mehr gekommen. Dass aber auch hier der eingeschlagene Weg, die Phosphortherapie, der richtige gewesen ist, beweisen eben die gefundenen Bilanzen. Wäre der Phosphor in einem früheren Stadium mit Ausdauer angewandt worden, so hätte gewiss der fortschreitende Kalkabbau und damit der hochgradige Kräfteverfall verhütet werden können.

Die beiden Versuche zeigen, dass der Kalkstoffwechsel der Osteomalacischen durch die Phosphortherapie günstig beeinflusst wird. Bei der ersten, später geheilten Patientin wird cine erhebliche Kalkretention erzielt, in dem zweiten, ungünstig verlaufenden Falle wird der weitere Kalkverlust beseitigt. Dieser Einfluss dauert aber nur so lange an, als das Medicament gegeben wird. Mit dem Aussetzen desselben tritt eine Kalkmehrausscheidung auf, so dass der Effect wieder ausgeglichen wird. Das Resultat dieses im kleinen durchgeführten Stoffwechselversuches ist durch den späteren Krankheitsverlauf bestätigt worden.

Im Folgenden soll eine Zusammenstellung bisheriger Stoffwechseluntersuchungen an Osteomalacischen gegeben werden. Tabelle IX giebt unter Anführung der betreffenden Autoren die Uebersicht über diese Resultate. Durch Umrechnung der Gesammtresultate verschieden langer Versuchsperioden auf den täglichen Durchschnittswerth dürften sich die einzelnen Befunde am besten vergleichen lassen.

Die ersten vollständigen Bilanzen sind 1894 von Limbeck und Neumann mitgetbeilt worden. Frühere Forscher haben wohl gründliche Harnanalysen vorgenommen; wir wissen aber durch von Noorden, dass gerade der Kalk zum weitaus grössten Theil durch den Darm ausgeschieden wird, und auch die Phosphorsäure verlässt, an Erdalkalien gebunden, in grosser Menge auf diesem Wege den Organismus. Die blossen Harnanalysen haben demnach nur einen bedingten Vergleichswerth. Maassgebend sind erst die vollkommenen Bilanzen derjenigen Autoren, welche einer genau berechneten Nahrungseinfuhr die Summe der Ausseheidungen in Koth und Urin gegenüberstellen.

R. v. Limbeck ${ }^{1}$ ) fand bei einer 56 jährigen Magd mit beginnender Osteomalacie neben einem durchaus normalen Stickstoffstoffwechsel eine beträchtlich gesteigerte Kalkausfuhr. Es muss jedoch berücksichtigt werden, dass die Kalkeinnahme eine sehr geringe gewesen ist.

Siegfried Neumann²) hat 1893 bei einer 37 jährigen Frau mit

1) R. v. Limbeck, Zur Kenntniss der Osteomalacie. Wien. med. Wochenschr. 1894. No. 17.

2) Siegfried Neumann, Quantitative Bestimmung des $\mathrm{Ca}, \mathrm{Mg}$ und der $\mathrm{P}_{2} \mathrm{O}_{5}$ im Harn und Koth bei Osteomalacie. Archiv f. Gynäk. 1894. Bd. 47. 


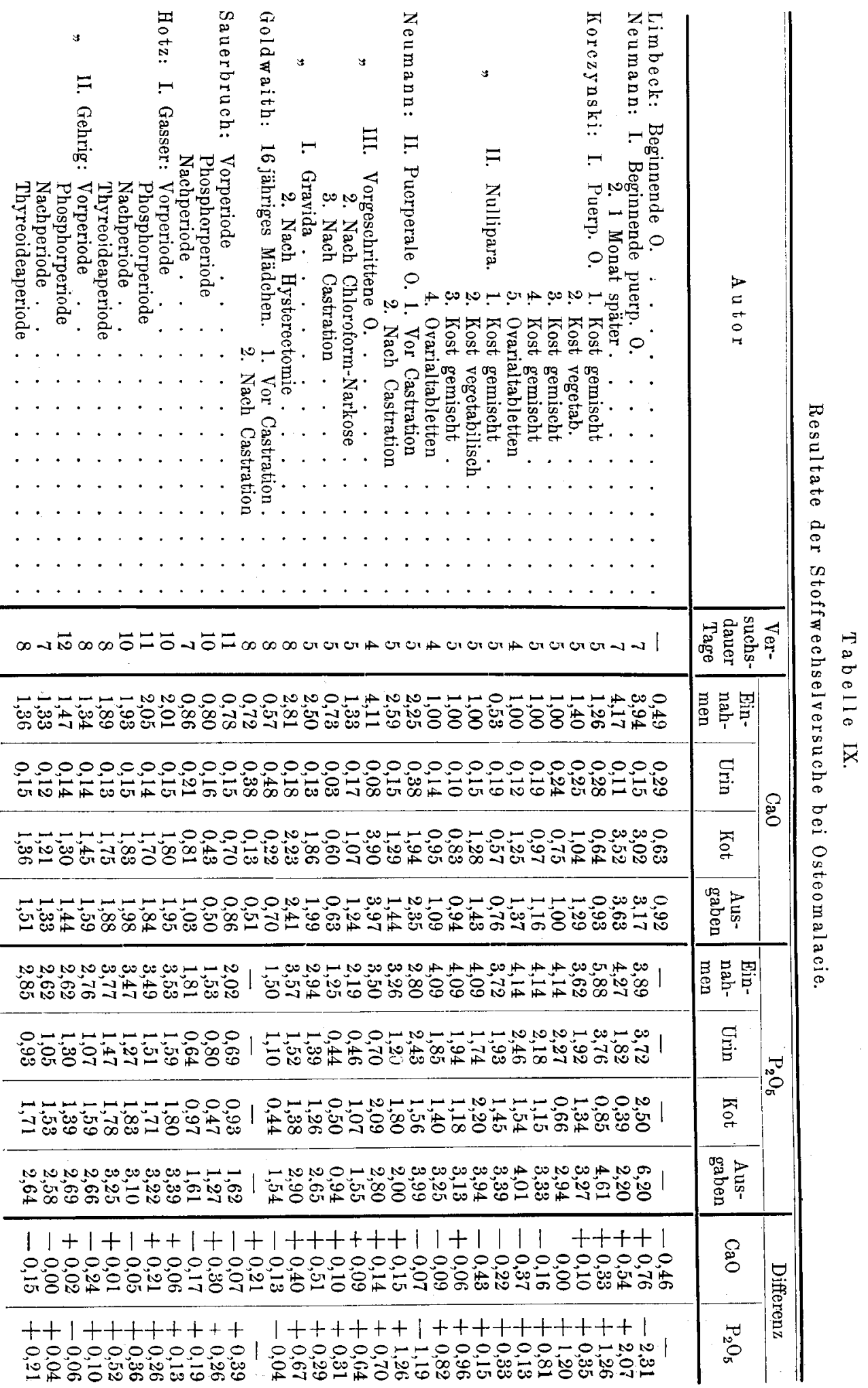


wenig vorgeschrittener Osteomalacie zwei Versuche angestellt, die sich über je sieben Tage erstrocken, und um einen Monat auseinanderliegen. Die Nahrung bestand aus 1,5-2,5 Iiter Milch und 300 cem Suppe täglich. Die Einnahme an $\mathrm{CaO}$ und $\mathrm{P}_{2} \mathrm{O}_{5}$ ist demgemäss eine grosse. Das erste Mal findet er eine Kalkretention von 0,76 und eine $\mathrm{P}_{2} \mathrm{O}_{5}$ Mehrausscheidung von 2,31 g. Das zweite Mal Retentionen: $\mathrm{CaO}=0,54$, $\mathrm{P}_{2} \mathrm{O}_{5}=2,07 \mathrm{~g}$. Auffallend ist die hochgradige Mehrausscheidung der $\mathrm{P}_{2} \mathrm{O}_{5}$ in der ersten Periode. Neumann glaubt, dass diese $15,18 \mathrm{~g}$ betragende Menge "in erster Reihe aus den Knochen, den an $\mathrm{P}_{2} \mathrm{O}_{5}$ reichsten Bestandtheilen des Organismus herstamme". Auf Grund dieses Befundes nimmt er an, dass sich die Frau damals im progressiven Krankheitsstadium befunden habe. Er stützt sich hierbei auch auf die Angabe der Krankengeschichte, wonach die Frau damals noch keiner Bewegung fähig war. Zur Zeit des späteren Versuches konnte sie sich dagegen schon ordentlich bewegen.

Die Möglichkeit, dass die $\mathrm{P}_{2} \mathrm{O}_{5}$-Bilanz wesentlich aus den Factoren des Eiweissstoffwechsels bedingt werde, ist nicht in Betracht gezogen. Auch wird nicht ausgeführt, weshalb Neumann bei der Beurtheilung des Krankheitsstadiums auf die Kalkbilanz verzichtet. Ich glaube, die Phosphorsäure kann aus verschiedenen Quellen stammen, auch aus anderen Organen, für Aenderungen am Aufbau der Knochen dagegen, giebt der Kalkstoffwechsel das beste Criterium. Halten wir uns an die Krankengeschichte dieses Falles. Die Frau hat sechs Wochen vor Beginn des ersten Versuches die neunte Entbindung durchgemacht. Im Laufe der siebenten Gravidität war die Osteomalacie zum Ausbruch gekommen. Nach der achten fühlte sie sich ebenfalls in ihren Bewegungen einige Wochen lang gehemmt. Jedes Mal trat später spontan die Besserung der Beschwerden ein - der typische Verlauf der puerperalen Osteomalacie. - Wir dürfen deshalb wohl annehmen, dass zur Zeit des Versuches, sechs Wochen nach der neunten Geburt, als der schädigende Einfluss der Gravidität abgeklungen war, sich die spontane Regeneration wieder einstellte. Dementsprechend finden wir eben einen grossen Kalkansatz. Die negative Differenz der $\mathrm{P}_{2} \mathrm{O}_{5}$ darf mit der Versuchsanordnung in Beziehung gebracht werden; denn die oben angeführte Kost kann nicht wohl als eine normale Ernährungsweise betrachtet werden.

In Wirklichkeit befindet sich die Patientin Neumann's bereits in der ersten Periode im reparatorischen Stadium, denn es findet eine Kalkretention statt. - - In therapeutischer Hinsicht empfiehlt Neumann die Verabreichung von Phosphorsäure, speciell die Milchdiät, „da die Milch, abgesehen von ihren übrigen Vorzügen für die Ernährung, in so grosser Menge $\mathrm{P}_{2} \mathrm{O}_{5}$ enthält, die, wie es scheint zum Ersatz jener $\mathrm{P}_{2} \mathrm{O}_{5}$ genügt, die während des früheren Stadiums der Krankheit verloren wurde".

Die Milch ist allerdings sehr zweckmässig für die Ernährung Osteomalacischer und zwar wegen ihres Kalkgehaltes, denn wie auch die Tabelle I zeigt, ist sie das einzige Nahrungsmittel, welches einigermaassen grosse Mengen organisch gebundenen Kalkes einführt, und eben des Calciums bedarf der Organismus zum Ausgleich des Knochenverlustes. 
Phosphorsäure geniessen wir in allen anderen Nahrungsmitteln, besonders im Fleisch und Brod zur Genügc.

Senator $\mathbf{r}^{1}$ ) bestimmte bei ciner 42 jährigen Frau die Stickstoff-, $\mathrm{CaO}$ - und $\mathrm{P}_{2} \mathrm{O}_{5}$-Ausscheidung unter dem Einfluss von Thyraden Knorr und Oophorin Freund. Die Nahrung war eine gleichmässige, wurde aber nicht der Analyse unterworfen. Er fand während der subjectiven und objectiven Besserung in der Oophorimperiode eine erheblich gesteigerte Kalkabgabe und glaubt daraus den.Schluss ziehen zu dürfen, dass auch ohne Abnahme der Kalkausfuhr eine Besserung der Osteomalacie eintreten könne. Dieser 11 tägigen Oophorinperiode liegt jedoch nur eine einzige Kothbestimmung, der am ersten und zweiten Tag ausgeschiedenen Menge und fünf Harnanalysen zu Grunde.

Korczynski²) führte an zwei Osteomalacischen fünf bezw. vier periodenweise angeordnete Untersuchungen aus. Bei der ersten Patientin handelt es sich um eine puerperale Form; der zweite Fall betrifft eine 20 jährige Nullipara. Die einzelnen Versuche umfassen je fünf Tage. In der ersten Periode wurde eine gemischte Kost mit ziemlich hohem $\mathrm{N}$ - und $\mathrm{P}_{2} \mathrm{O}_{5}$-Gehalt gegeben; in der zweiten vegetabilische Kost mit unbedeutender $\mathrm{N}$-, mittlerer Phosphor- und hoher Kalkmenge; die dritte und vierte entspricht einer Nahrungsweise von gewöhnlicher Zusammenstellung; während der letzten wurden bei gleicher Kost sechs bis neun Ovarialtabletten à $0,25 \mathrm{~g}$ Substanz gegeben. Eine Besserung der Krankheit wurde während der Versuchszeit bei keiner der beiden Personen beobachtet.

Im ersten Fall zeigt die Kalkausscheidung im Harn anfänglich normale Werthe, sinkt aber in der vierten und fünften Periode auf sehr geringe, subnormale Mengen. Zugleich steigt der Kalkgehalt des Kothes, und dadurch wird die ursprünglich positive CaO-Bilanz in eine negative umgewandelt.

Für den zweiten Fall ist die Kalkbilanz mit Ausnahme einer geringen Retention in der dritten Periode stets negativ, der Urinkalk steht unter der Norm. Phosphorsäure wird in allen Versuchen zurückgehalten. Das normale Verhältniss der $\mathrm{P}_{2} \mathrm{O}_{5}$-Ausscheidung in Harn und Koth hat eine Aenderung erfahren. Während bei Gesunden ïber 80 pCt. des Phosphors im Urin abgegeben werden, sinkt hier die Menge bis auf 50 pCt der Tagesausscheidung. Daraus ergiebt sich die Thatsache, dass Kalk- und Phosphorabgabe einander parallel gehen. Eine Verminderung der Kalkmenge im Urin wird von einer geringeren Phosphorsäuresecretion begleitet, gleichzeitig steigt aber die Abgabe der beiden durch den Darm. Korezynski glaubt, aus seinen Befunden auf eine Prognose schliessen zu dürfen. Wenn nämlich im Harn normale Phosphor- und Kalkquantitäten gefunden werden, soll die Krankheit zur Besserung und zur Ausheilung neigen; wenn aber die anormalen Verhältnisse längere Zeit

1) H. Senator, Zur Kenntniss der Osteomalacie und der Organotherapie. Berl. klin. Wochenschr. 1897. No. 6.

2) L. R. v. Korezynski, Zur Kenntniss des Stoffwechsels bei Osteomalacie. Wiener med. Presse. 1902. S. 1073. 
bestehen, scheint die Prognose eine ungünstige zu sein. Er empfiehlt dann als Ultimum refugium die Castration.

Dies führt uns zu den Stoffwechselversuchen, welche zur Feststellung therapeutischer Einflüsse auf die Osteomalacie vorgenommen wurden.

Siegfried Neumann ${ }^{1}$ ) hat in dieser Weise bei 3 Frauen den Erfolg der Castration studirt. Der erste Fall zeigt uns eine 36 jährige Kranke, bei welcher das Leiden nach der sechsten Geburt aufgetreten war. Da während der folgenden 6 Monate trotz guter Spitalpflege, jedoch ohne specielle Therapie, sich keine Besserung zeigte, wurde die Castration ausgeführt. Daraufhin genas die Patientin. Die erste Untersuchung fand 45 Tage vor, die zweite 20 Tage nach der Operation statt; jede erstreckte sich über 5 Tage. In der ersten Periode fand er einen geringen Kalkverlust, nach der Castration einen sehr grossen Kalkansatz von $1,15 \mathrm{~g}$ pro Tag. Daraus geht hervor, dass die radicale Therapie in diesem Falle den Kalkstoffwechsel in der günstigsten Weise beeinflusst hat. Das Gleiche gilt auch für die Phosphorsäure.

Der zweite Fall betrifft eine 36 jährige, bereits kachektische Frau mit hochgradigen Knochenveränderungen. Der erste Versuch wurde 50 Tage vor, der zweite 8 Tage nach einer längeren Chloroformnarkose vorgenommen, um den Einfluss dieser, von Petrone empfohlenen Therapie zu bestimmen. Da sich klinisch keinerlei Einwirkung auf den Krankheitsverlauf constatiren liess, wurde nach Kurzem die Castration ausgeführt und 5 Tage später eine neue Versuchsperiode angeschlossen. Die beiden ersten Bilanzen ergaben eine geringe Kalkretention, welche durch die Chloroformnarkose nicht verändert wird, auch die Castration bewirkt keinen Kalkansatz, allerdings auch keine Besserung. Die Phosphorsäureretention geht während der ganzen Zeit langsam zurück. Auf Grund dieses Befundes glaubt N., dass der osteomalacisch erkrankte Organismus nur bis zu einer gewissen Grenze $\mathrm{CaO}$ und $\mathrm{P}_{2} \mathrm{O}_{5}$ verlieren könne, später vollziehe sich eine geringe Retention dieser Stoffe; jedoch ohne dass dadurch die früheren Verluste gedeckt werden könnten.

Der dritte Fall giebt die Fortsetzung der in der ersten Publication Neumann's erwähnten Versuche von 1893. Ein Jahr lang hatte sich die Frau nach ihrem Austritte gesund gefühlt, dann wurde sie von Neuem gravid und damit war die Veranlassung zum Wiederaufflackern der Osteomalacie gegeben. Als Gravida im fünften Monat kam sie wieder zur Spitalaufnahme. Da die Krankheit stürmische Fortschritte machte, wurde die Hysterectomie des graviden Uterus mit Entfernung der Adnexe ausgeführt. Die Heilung setzte sofort ein und anderthalb Jahre später erfreute sich die Frau einer vollkommenen Arbeitsfähigkeit. Die beiden Versuche wurden 8 Tage vor, bezw. 20 Tage nach der Operation angestellt. Zur Zeit der Schwangerschaft wurde ein Kalkansatz von $0,5 \mathrm{~g}$

1) Siegfried Neumann, Weitere Untersuchungen über die Stoffwechselverhältnisse des $\mathrm{Ca}, \mathrm{Mg}, \mathrm{P}_{2} \mathrm{O}_{5}$ und Nitrogens bei puerperaler Osteomalacie, mit besonderer Rücksicht auf die durch Castration verursachten Veränderungen. Archiv $\mathrm{f}$. Gynäk. Bd. 51. 1896. 
gefunden. Der Organismus hat im Vergleich zu der früheren, ersten Retention von $0,76 \mathrm{~g}$ weniger $\mathrm{CaO}$ zurïckgehalten. Neumann folgert daraus, dass der Kalkbedarf der Frucht mindestens theilweise aus dem Vorrath des mütterlichen Körpers gedeckt worden sein müsse. Es scheint mir jedoch keineswegs bewiesen, dass ein täglicher Kalküberschuss von $0,5 \mathrm{~g} \mathrm{CaO}$ zur Entwicklung eines 5 monatigen Fötus nicht genüge. Die Gewichtszunahme eines Fötus beträgt vom fünften bis zum sechsten Monat etwa $400 \mathrm{~g}$. Aschenanalysen solcher Früchte sind wohl bisher nicht ausgeführt worden. Den Aschengehalt von Neugeborenen bestimmten Camerer und Söldner ${ }^{1}$ ) auf 2 pCt., darunter $\mathrm{CaO} 9,0$ auf $100 \mathrm{~g}$ Asche. Berechnen wir an Hand dieser Zahlen den Kalkansatz des menschlichen Fötus zwischen dem fünften und sechsten Monat, so finden wir für 30 Tage nur eine Zunahme von $0,8 \mathrm{~g} \mathrm{CaO}$, eine Menge, die jedenfalls durch die von Neumann constatirte Retention von $0,5 \mathrm{~g}$ pro Tag reichlich geliefert werden kann, ohne dass "der mütterliche Vorrath" angegriffen werden müsste.

Nach der Hysterectomie wurden täglich $0,4 \mathrm{~g}$ Kalk retinirt. Neumann glaubt, dass die Regeneration der Knochen zu dieser Zeit schon beendet sein müsste, da die Patientin sich bereits gesund fühlte und keiner grösseren Kalkmengen mehr bedurfte. Die Phosphorsäure ergiebt nach der Castration gesteigerten Ansatz. Zum Schlusse fasst Neumann seine Untersuchungen folgendermaassen zusammen:

Die Kalkausscheidung im Urin ist zu Beginn der Krankheit am grössten; später nimmt sie in Folge der Decalcination allmählich ab. Die anfänglich grossen Kalkverluste finden später eine Grenze; der Organismus hält den Rest zähe zurück. Hat der Körper in kurzer Zeit viel Kalk verloren, so vermag er denselben sehr rasch wieder zu ersetzen. Die operative Therapie vermag den Stoffwechsel in der günstigsten Weise zu beeinflussen; aber die Castration hat nur bis zu gewissen Grenzen ihre Berechtigung. In sehr vorgeschrittenen Fällen kann sie schaden.

$\mathrm{Zu}$ ähnlichen Ansichten kommen auch Goldwaith, Osgood und Rudden ${ }^{2}$ ). Diese haben bei einem 16 jährigen Mädchen mit sehr verunstaltetem Skelett zwei Bestimmungen von 8 und 14 Tagen ausgeführt. Sie fanden vor der Castration, bei allerdings geringer Nahrungszufuhr, einen Calcium- und Phosphorsäureverlust, einige Monate nach der Operation eine Kalkretention von $0,2 \mathrm{~g}$ täglich. Die zweite Phosphorsäurebestimmung fehlt hier.

Die jüngste Arbeit zur Feststellung therapeutischer Maassnahmen gegen Osteomalacie, welche gewissermaassen die Veranlassung zu den vorliegenden Untersuchungen gegeben hat, bildet die Abhandlung von F. Sauerbruch ${ }^{3}$ ), welcher den Einfluss der Phosphortherapie bestimmte.

1) Citirt bei Albu and Neuberg, Physiologie und Pathologie des Mineralstoffwechsels. Berlin. 1906. S. 25.

2) A study of the metabolism in osteomalacia. The american journal of physiol. Bd. 14. (1905.) S. 389.

3) F. Sauerbruch, Ein Beitrag zum Stoffwechsel des Kalks und der Phosphorsäure bei infantiler 0steomalacie. Inaug,-Diss. Leipzig. 1902. 
Es handelt sich um einen 8 jährigen Jungen, der seit mehr als 2 Jahren an juveniler Osteomalacie leidet, welche sich durch zahlreiche Fracturen und Verbiegungen des Skelettes äussert. Der Kranke erhält Phosphorleberthran. Allgemeinbefinden und Gewichtszunahme sind befriedigend, aber die Knochenbrüchigkeit blieb bestehen. Die Versuchsanordnung ist cine ähnliche wie für unsere Fälle - Vor-, Phosphor- und Nachperiode. Für die Vor- und Nachperiode ergab sich ein durchschnittlicher Kalkverlust von 0,07 resp. $0,17 \mathrm{~g}$ pro Tag. Während der Phosphordarreichung wurde ein täglicher Ansatz von $0,3 \mathrm{~g}$ beobachtet. Dieses auffallende Resultat schreibt $\mathrm{S}$. der Wirkung der Phosphortherapie zu. Die $\mathrm{P}_{2} \mathrm{O}_{5}$ Bilanz erfährt dagegen keine bemerkenswerthe Aenderung. Sowohl in der Vor- wie in der Nachperiode findet eine Retention statt, 0,39 resp. 0,19 g. Während der Phosphoreinwirkung ergiebt sich ein mittlerer Ansatz von $0,25 \mathrm{~g}$.

Gegen Sauerbruch's Arbeit liesse sich vielleicht einwenden, dass die Angaben darüber fehlen, wie der Koth abgegrenzt und für die einzelnen Tagesausgaben berechnet wurde. $\Lambda \mathrm{m}$ 9. März und am 15. März, an 2 Tagen seiner Phosphorperiode, gingen die Gesammtausscheidungen verloren. Indem nun auch die Einnahmen für die Gesammtberechnung weggelassen wurden, sind die Verlusttage ganz aus der Bilanz ausgeschaltet worden; es konnte aber mit dem Koth eine grössere Menge weggegossen worden sein, welche eigentlich für die Bilanz der vorherigen Tage in Betracht zu ziehen wäre. Ausserdem ist nicht zu ersehen, wie S. für $200 \mathrm{~g}$ Milch eine Kalkeinfuhr von $0,639 \mathrm{~g} \mathrm{CaO}$ pro Tag findet. Seine Tabelle der Nahrungsmittelanalysen ergiebt für $100 \mathrm{~g}$ die Werthe 0,161 bis $0,191 \mathrm{~g} \mathrm{CaO}$.

Den in Tabelle IX aus der mir bekannten Literatur gesammelten Zahlen füge ich zum Vergleich die Resultate der eigenen Beobachtungen bei. Aus diesen Erfahrungen dürften etwa folgende Schlüsse über den Ablauf des Kalk- und Phosphorstoffwechsels zu ziehen sein.

Was den Kalk anbetrifft, so muss zur Beurtheilung der Frage, ob eine pathologische Mehrausscheidung vorliege, vor Allem eine genügend grosse Einnahme an resorbirbarem Calcium garantirt sein, denn auch beim Gesunden findet, wie Renvall ${ }^{1}$ ) gezeigt hat, eine negative Kalkbilanz statt, sobald die zugeführte Menge ein gewisses Minimum nicht erreicht. Renvall berechnet den täglichen Kalkbedarf des Erwachsenen auf $0,6 \mathrm{~g}$, Bertram $^{2}$ ) auf $0,4 \mathrm{~g} \mathrm{CaO}$. Unter den zusammengestellten 32 Versuchsperioden finden wir 4 Mal kleinere Worthe, nämlich bei Korczynski's 2. Fall im ersten Versuch, bei Neumann's 2. Castrationsfall nach der Operation, bei Goldwaith's Versuchen. Unter solchen Bedingungen ist ein Kalkverlust wohl erklärlich; und wenn Neumann bei einer Einnabme von $0,73 \mathrm{~g}$ noch eine kleine Retention von $1 \mathrm{deg}$ erzielt, so spricht dies eigentlich nur dafür, dass

1) G. Renvall, Zur Kenntniss des P-, Ca-, Mg-Umsatzes beim Erwachsenen, Scand. Arch. f. Physiologie. Bd. 16. S. 94.

2) Bertram, Zeitschr. f. Biologie. Bd. 14. 1878. 
bei vorhandenem Material wohl grössere Mengen assimilirt worden wären, also für den Erfolg der Castration. In den Versuchen mit grosser Kalkzufuhr treffen wir stets einen Ansatz, einzig beim ersten Neumann'schen Castrationsfall im floriden Krankheitsstadium vor der Operation und während der Nachperiode bei Frau Gasser tritt geringe Mehrausscheidung auf. Jedenfalls steht so viel fest, wollen wir bei Osteomalacie durch irgend welche Therapie eine Knochenfestigung erreichen, so muss kalkreiche Nahrung gegeben werden, und diese Bedingung erfüllt am besten die Milch.

Die Kalkausscheidung vollzieht sich in Harn und Koth. Die täglichen Urinkalkmengen beim Gesunden. betragen nach Beckmann 0,5 g, Bunge $0,3 \mathrm{~g}$, Schetelig $0,3-0,5 \mathrm{~g}$, Renvall $0,5 \mathrm{~g} \mathrm{CaO}^{1}$ ). In Tabelle IX finden wir fast durchweg sehr geringe Mengen von $1-2 \mathrm{deg}$, die angeführten Normalzahlen werden kaum erreicht, jedenfalls nie überschritten, und in den Fällen, welche einer mehrmaligen Bestimmung unterworfen wurden, ändert sich die Urinausscheidung nicht wesentlich, gleichgültig, ob negative oder positive Kalkbilanz auftritt. Nur bei Neumann sinkt nach der Castration bei sehr grosser Kalkretention der Urinwerth auf die Hälfte. Eine Steigerung oder Verminderung des Kalkes in der Nahrung bleibt ohne Einfluss auf die Nierensecretion. Es steht also fest, dass die Harnsecretion sich nicht an der Halisteresis betheiligt. Die seiner Zeit von Schuchard angegebene und seither in verschiedene Arbeiten übergegangene Behauptung, dass in den Anfangsstadien der Krankheit die Harnkalkausscheidung weit über die Norm vermehrt sei, dürfte deshalb wohl bezweifelt werden.

Der Kalk verlässt den Körper auf dem Darmwege. Wir sehen gerade aus den eigenen Fällen, dass die geringste Aenderung der Kalkbilanz durch entsprechende Schwankungen im Kothgehalt verursacht wird, auch die übrigen Versuche illustriren dieses Verhalten, wenn nicht durch grössere Differenzen in der Einnahme dieses Bild gestört wird. Je höher die Kothausscheidung, desto geringer wird der Harngehalt. Es scheint, dass der Organismus bestrebt sei, auf der einen Seite zurückzuhalten, was er auf der anderen zu viel ausgiebt. Dies zeigt sich besonders deutlich in Korczynski's Versuchen, wo beim Auftreten der negativen Bilanz nur sehr niedrige Mengen im Urin ausgeführt werden. Sobald andererseits die Krankheit in das Stadium der Regeneration tritt, sei es durch verbesserte hygienische und Ernährungsverhältnisse oder durch therapeutische Einflüsse verringert sich nur die Menge des Kothkalkes.

Die Phosphorsäure schlägt im Körper 2 Wege ein. Der grössere Theil wird durch den Harn ausgeschieden. Die Quantität der $\mathrm{P}_{2} \mathrm{O}_{5}$-Secretion durch die Nieren wird, im directen Gegensatz zum Kalk, in erster Linie von der Nahrungsaufnahme bestimmt. Bei gesteigerter Zufuhr von animalischem Eiweiss, Nucleinen etc. wächst die Harnmenge, zweitens kommt der Phosphorsäureansatz in Betracht. Es ist auffallend, dass beinahe in allen Versuchen eine $\mathrm{P}_{2} \mathrm{O}_{5}$-Retention constatirt wurde,

1) Angeführt bei Renvall, l. c. 
welche, wie z. B. im Falle Gasser in steter Zunahme begriffen ist. Eine Mehrausscheidung treffen wir bei 2 Patienten Neumann's, bei Goldwaith und während der Phosphorperiode der Frau Gehrig. Der osteomalacische Organismus hat augenscheinlich die Tendenz, erhebliche Mengen von Phosphorsäure aufzuspeichern. Diese Anreicherung begleitet in mehreren Fällen die klinische Besserung, ist bei Frau Gasser mit einer erheblichen Gewichtszunahme verbunden. Drittens wird die Urinausscheidung durch die Darmabgabe beeinflusst, welche normaler Weise erheblich niedriger als die Nierensecretion, bei Osteomalacischen unter dem Einfluss der vermehrten Kalkausfuhr, die Harnabgabe übertreffen kann. Diese 3 Factoren: Nahrungsaufnahme, Ansatz im Organismus und Darmausscheidung können sich in der verschiedensten Weise combiniren, so dass auch durch Beiziehen der Stickstoffbestimmung ( $\mathrm{Neu}$ mann, Limbeck, Korczynski) ein einheitliches Urtheil über die Schicksale der Phosphorsäure nicht zu gewinnen ist.

Die Phosphorsäureabgabe durch den Darm ist, wie Korczynski gezeigt hat, relativ vermehrt. Statt einer Ausscheidung von 20 pCt. fand er im Koth bis 50 pCt. (cf. S. 27). Unser erster Fall bietet die Eigenthümlichkeit, dass der $\mathrm{CaO}$ - und $\mathrm{P}_{2} \mathrm{O}_{5}$-Gehalt in den Fäces genau übereinstimmen, d. h. es wird in Grammen genau so viel $\mathrm{CaO}$ wie $\mathrm{P}_{2} \mathrm{O}_{5}$ abgegeben und die dureh die Versuchsanordnung bedingten Schwankungen äussern sich bei beiden Elementen durch gleiche Gewichtsdifferenzen. Diese Erscheinung treffen wir, wenn auch nicht in so eclatanter Deutlichkeit, bei den Versuchen der Autoren wieder. Steigt die absolute Kalkmenge im Koth, so wird auch mehr Phosphorsäure abgegeben. Diese Beobachtung finden wir in 15 von 18 Vergleichsversuchen bestätigt. Nur 3 Mal besteht ein gegentheiliges Verhalten, 2 Mal bei Neumann und in der Nachperiode von Frau Gehrig. Trotz dieser Ausnahme ergiebt sich im Allgemeinen die Thatsache, dass der Phosphorsäuregehalt des Kothes in enger Beziehung steht zu dessen Kalkmenge, ja es scheint, dass er sich nur nach der durch den Darm ausgeschiedenen Quantität des Calciums richtet. Er begleitet in verschiedenen Versuchen die Kalkschwankungen, aber in Berücksichtigung der noch wenig bekannten Factoren des Phosphorstoffwechsels. Nahrungsaufnahme, Körperansatz, Harn- und Kothausscheidung, lässt sich bis jetzt nicht feststellen, welcher Natur dieser Zusammenhang eigentlich ist.

Es bestehen 2 Möglichkeiten. Entweder, die Base, der Kalk wird primär ausgeschieden und reisst die Phosphorsäure gewissermaassen mit sich; oder, die Phosphorsäure wird in übernormaler Menge frei und verbindet sich im Organismus mit dem Kalk, welcher den Knochen entzogen wird.

Die Säuretheorie spielt in der Aetiologie der Osteomalacie eine grosse Rolle. Erst wurde eine pathologische Bildung von Milchsäure, dann von Kohlensäure, schliesslich von Oxalsäure angenommen, welche den Kalk der Knochen auslangen sollte. An die Phosphorsäure ist meines Wissens nicht gedacht worden. Nun finden wir im Koth eine vermehrte Ausscheidung von Kalk, welche, wie oben gezeigt wurde, Hand in Hand geht mit einer grösseren Abgabe von $\mathrm{P}_{2} \mathrm{O}_{5}$. Es liegt 
nun sehr nahe, die Kalkausscheidung, dic wir als sicheren Indicator der Halisterese betrachten dürfen, der Ammoniakausscheidung beim Diabetes resp. bei der Acidosis als Analogon an die Seite zu stellen. Wie das $\mathrm{NH}_{3}$ im Harn durch die Anwesenheit der $\beta$-Oxybuttersäure bedingt ist, und zur Beurtheilung dieser Acidosis dient, so könnte die Kalkausscheidung im Darm auf eine andere Acidosis, eben auf vermehrie Phosphorsäure hinweisen. Diese Theorie dürfte vielleicht durch das Experiment bewiesen werden, wenn es gelingt, nach künstlicher Einverleibung von Phosphorsäure eine negative Kalkbilanz, eine Mehrausgabe durch den Darm hervorzurufen.

Jedenfalls ist die Phosphorsäure kein Indicator zur Beurtheilung, in welchem Stadium sich eine Osteomalaciekranke befindet. Die Kalkbilanz dagegen lässt dies erkennen. Finden wir hier einen Verlust, so besteht Halisteresis. Der Stillstand des Processes und die Regeneration werden durch eine positive Kalkbilanz bewiesen. Der Kalkansatz ist um so grösser, je rascher die Genesung fortschreitet. So bei Neumann's günstig verlaufenden Fällen, bei Sauerbruch und bei unserer ersten Kranken. Wo aber bereits eine Jahre lange Kalkberaubung am Skelett gezehrt hat, so dass Kachexie und schlechte Assimilation der Nahrung aufgetreten ist, da wird sowohl der zahlengemässe Kalkansatz, wie auch die subjective Besserung langsamer einsetzen.

Die Kalkbilanz belehrt uns über den Werth der eingeschlagenen Therapie.

In manchen Fällen ergiebt die Krankenhausbehandlung an sich mit ihrem günstigen Einfluss auf die Ernährungsverhältnisse und den Energieverbrauch bereits eine sichtliche Besserung; erfahrungsgemäss bei puerperalen Fällen nach der Geburt.

Insbesondere interessiren jedoch die specifisch therapeutischen Einflüsse, zunächst die Organotherapie. Bei der Verabreichung von Ovarialtabletten findet Korczynski beide Male negative Bilanzen. Die Thyreoideatabletten, welche bei unseren beiden Frauen im Anschluss an die übrigen Versuche gegeben wurden, lassen eine besondere Einwirkung der Schilddrüsensubstanz auf den Kalkstoffwechsel nicht erkennen. Immerbin sollen aus diesen 2 Fällen keine bindenden Schlüsse über die Thyreoideatherapie gezogen werden, hierzu müsste ebenfalls eine Anordnung von 3 Versuchsperioden getroffen werden. Wir betrachten diese 8 Tage vielmehr als eine einfache Verlängerung der Nachperiode, während weleher der vorausgegangene Einfluss des Phosphors und seine Entziehung langsam abklingen.

Die Chloroformnarkose nach Petrone ist auf Kalk- und Phosphorbilanz ohne Einfluss.

Nun stehen sich noch zwei gleich erprobte Massnahmen gegenüber: Die von Fehling eingeführte radicale Therapie, die Castration und die conservative, die Phosphorbehandlung. Es würde zu weit führen, das klinische Material zu berücksichtigen. Aus der gegebenen Zusammenstellung von Stoffwechselversuchen illustriren 4 den Einfluss der Castration.

Der zweite Fall von Neumann wird durch die Operation geheilt. Statt eines Deficites vorher tritt eine grosse Retention von 1,15 g CaO 
pro Tag auf. Sein dritter, bereits weit vorgeschrittener Fall wird nicht gebessert; der anfänglich geringe Kalkansatz sinkt weiter von 0,14 auf $0,10 \mathrm{~g}$. Die erste Kranke kommt nach Entfernung des graviden Uterus mit den Adnexen zur Genesung; es besteht eine positive Kalkbilanz, Goldwaith erzielte durch die Castration ebenfalls einen günstigen Verlauf und verzeichnet an Stelle der Mehrausscheidung einen erheblichen Kalkgewinn.

Die Stoffwechselversuche bestätigen somit die klinischen Erfahrungen der operativen Therapie.

Nicht alle Fälle werden geheilt; sehr vorgeschrittene bleiben öfters unbeeinflusst; aber wenn die Krankheit in das Stadium der Regeneration tritt, wird sie von Kalkansatz begleitet.

Dem radicalen Vorgehen ist die Phosphortherapie an die Seite zu stellen. Im Sauerbruch'schen Falle wird die bestehende Halisteresis durch die Einwirkung des Phosphoröls in eine wesentliche Kalkretention umgewandelt.

Durch die eigenen Untersuchungen glaube ich den günstigen Einfluss des Phosphors auf den Kalkstoffwechsel bei Osteomalacischen nachgewiesen zu haben; denn bei der ersten Frau führt diese Therapie zu einem gesteigerten Kalkansatz, zur Heilung, bei der Anderen setzt sie dem stetigen grossen Kalkverlust eine Grenze und unterbricht den weiteren Fortschritt des Leidens. Es zeigt sich aber, dass die Phosphortherapie nur während der Zeit ihrer Anwendung wirksam ist, setzt man das Medicament nach Kurzem aus, so wird der Effect durch eine Mehrausscheidung wieder ausgeglichen. Nur eine über lange Zeit fortgesetzte Phosphorbehandlung führt zu einem dauernden Resultat.

Darüber, wie der Phosphor eigentlich auf die Knochensubstanz einwirkt, besteht gegenwärtig noch keine Erklärung: den einzigen sicheren Anhaltspunkt giebt uns Wegner ${ }^{1}$ ), welcher nach Darreichung von anorganischem Phosphor bei neugeborenen Kaninchen charakteristische Veränderungen der Knochen auftreten sah. Er berichtet darüber: „Unter dem Einfluss der Phosphorfütterung wird an allen den Stellen, wo sich aus Knorpel physiologisch spongiöse Substanz entwickelt, statt dessen ein Gewebe erzeugt, das bei blosser Betrachtung wie die Rinde von Röhrenknochen, also wie Compacta aussieht, am intensivsten um die Kerne der Epiphyse und Diaphyse der Oberschenkel. Hier bildet es einen dichten, weissen Saum statt der weitmaschigen, rothes Mark enthaltenden Spongiosa. Die Substanz erweist sich mikroskopisch als wirklicher wohlgebildeter Knochen. Die Markräume sind reducirt bis auf die Weite der Havers'schen Canäle in der gew öhnlichen Compacta, indem die Knorpelzellen nicht in Markzellen, sondern in Knochenkörperchen umgewandelt werden. Setzt man die Phosphorfütterung weiter fort, so wird von dem Intermediärknorpel der Röhrenknochen immer weiter verdichtetes Gewebe apponirt, während die vorhandene Spongiosa nach physiologischem Gesetz zur Bildung der Markhöhle eingeschmol\%en wird. Ordnet man

1) Wegner, Der Einfluss des Phosphors auf den Organismus. Virchow's Arch. f. path. Anat. u. Physiol, 1872. Bd. 55. 
eine Phosphorfütterung mit Intervallen an, so finden sich abwechselnd Schichten von verdichteter, compacter und weitmaschiger spongiöser Substanz.

Nach vollendetem Wachsthum wird bei Phosphordarreichung die Spongiosa der Epiphysen und der Wirbel wohl dichter, die Bälkchen breiter und dicker, aber es bildet sich nicht annähernd die Sklerose wie beim wachsenden Thier. Nach Wegner resultirt die Phosphorwirkung nicht aus den Oxydationsproducten, sondern sie beruht auf einem formativen Reiz specifischer Art, den der im Blut circulirende Phosphor auf die osteogenen Gewebe ausübt.

Mit dieser Darstellung, welche an Präcision nichts zu wünschen übrig lässt, müssen wir uns vor der Hand begnügen. In wiefern der pathologisch veränderte, speciell der osteomalacische Knochen, bei welchem Resorption und Apposition die grösste Rolle spielen, einer solchen "Sklerose" fähig ist, ist durch anatomische Untersuchungen nicht aufgeklärt. Der klinische Verlauf und die Stoffwechselversuche unter dem Einfluss der Phosphortherapie lassen etwas Achnliches vermuthen.

Aus diesen Erfahrungen möchte ich aber noch weitere therapeutische Schlüsse ziehen.

Liesse sich diese, das Knochengewebe verdichtende Eigenschaft des Phosphors nicht auch gegen andere pathologische Processe anwenden, bei welchen die Knochenbildung gestört ist oder eine krankhafte Einschmelzung stattfindet? Die günstige Wirkung der Phosphoremulsion bei Rhachitis ist ja allbekannt; es möchte sich vielleicht empfehlen, den Versuch der Phosphorbehandlung auszudehnen auf schlecht consolidirte Fracturen und namentlich auf die Tuberculose. 